\title{
One-cohomology and the uniqueness of the group measure space decomposition of a $\mathrm{II}_{1}$ factor
}

\author{
By StefaAn VAe: $(1) \mid(2)$ \\ Mathematische Annalen 355 (2013), 661-696.
}

\begin{abstract}
We provide a unified and self-contained treatment of several of the recent uniqueness theorems for the group measure space decomposition of a $\mathrm{II}_{1}$ factor. We single out a large class of groups $\Gamma$, characterized by a one-cohomology property, and prove that for every free ergodic probability measure preserving action of $\Gamma$ the associated $\mathrm{II}_{1}$ factor has a unique group measure space Cartan subalgebra up to unitary conjugacy. Our methods follow closely a recent article of Chifan-Peterson, but we replace the usage of Peterson's unbounded derivations by Thomas Sinclair's dilation into a malleable deformation by a one-parameter group of automorphisms.
\end{abstract}

\section{Introduction and main results}

A fundamental problem in the theory of von Neumann algebras is the classification of group measure space $\mathrm{II}_{1}$ factors $\mathrm{L}^{\infty}(X) \rtimes \Gamma$ in terms of the initial free ergodic probability measure preserving (pmp) action $\Gamma \curvearrowright(X, \mu)$. This problem breaks up in two very different parts. Given an isomorphism $\mathrm{L}^{\infty}(X) \rtimes \Gamma \cong \mathrm{L}^{\infty}(Y) \rtimes \Lambda$ one first studies whether $\mathrm{L}^{\infty}(X)$ and $\mathrm{L}^{\infty}(Y)$ are unitarily conjugate. If so this implies that the orbit equivalence relations $\mathcal{R}(\Gamma \curvearrowright X)$ and $\mathcal{R}(\Lambda \curvearrowright Y)$ are isomorphic, leading to the second problem of classifying group actions up to orbit equivalence. This paper deals with the first of these two problems: the uniqueness up to unitary conjugacy of the Cartan subalgebra $\mathrm{L}^{\infty}(X) \subset \mathrm{L}^{\infty}(X) \rtimes \Gamma$.

A Cartan subalgebra $A$ of a $\mathrm{II}_{1}$ factor $M$ is a maximal abelian von Neumann subalgebra for which the group of unitaries normalizing $A$, i.e. $\left\{u \in \mathcal{U}(M) \mid u A u^{*}=A\right\}$, generates the whole of $M$. If $\Gamma \curvearrowright(X, \mu)$ is a free ergodic pmp action, then $\mathrm{L}^{\infty}(X) \subset \mathrm{L}^{\infty}(X) \rtimes \Gamma$ is a Cartan subalgebra. Not all Cartan subalgebras in a $\mathrm{II}_{1}$ factor can be realized in this way. If they can, we call them group measure space Cartan subalgebras.

By [CFW81] the hyperfinite $\mathrm{II}_{1}$ factor $R$ has a unique Cartan subalgebra up to conjugacy by an automorphism of $R$. Until recently no other uniqueness theorems for Cartan subalgebras were known. A first breakthrough was realized by Ozawa and Popa in [OP07] who proved that the $\mathrm{II}_{1}$ factors $M=\mathrm{L}^{\infty}(X) \rtimes \Gamma$ coming from profinite free ergodic pmp actions of a direct product of free groups $\Gamma=\mathbb{F}_{n_{1}} \times \cdots \times \mathbb{F}_{n_{k}}$, have a unique Cartan subalgebra up to unitary conjugacy. In a second article [OP08. Ozawa and Popa establish the same result for all profinite actions of groups $\Gamma$ satisfying the complete metric approximation property and a strong form of the Haagerup property. This includes lattices in direct products of $\mathrm{SO}(n, 1)$ and $\mathrm{SU}(n, 1)$. Peterson then showed in [Pe09] that $M=\mathrm{L}^{\infty}(X) \rtimes \Gamma$ has a unique group measure space Cartan subalgebra whenever $\Gamma \curvearrowright(X, \mu)$ is a profinite action of a free product $\Gamma_{1} * \Gamma_{2}$ where $\Gamma_{1}$ does not have the Haagerup property and where $\Gamma_{2} \neq\{e\}$.

\footnotetext{
${ }^{(1)}$ Partially supported by ERC Starting Grant VNALG-200749, Research Programme G.0639.11 of the Research Foundation - Flanders (FWO) and K.U.Leuven BOF research grant OT/08/032.

${ }^{(2)}$ Department of Mathematics; University of Leuven; Celestijnenlaan 200B; B-3001 Leuven (Belgium). E-mail: stefaan.vaes@wis.kuleuven.be
} 
In the joint article [PV09] with Sorin Popa, we introduced a family of amalgamated free product groups $\Gamma=\Gamma_{1} *_{\Sigma} \Gamma_{2}$ such that for every free ergodic pmp action $\Gamma \curvearrowright(X, \mu)$, the $\mathrm{II}_{1}$ factor $M:=\mathrm{L}^{\infty}(X) \rtimes \Gamma$ has a unique group measure space Cartan subalgebra up to unitary conjugacy. The family of groups covered by [PV09] consists of the amalgamated free products $\Gamma=\Gamma_{1} *_{\Sigma} \Gamma_{2}$ such that $\Sigma$ is amenable and weakly malnorma $\sqrt{(3)}$ in $\Gamma$ and such that $\Gamma$ either admits a nonamenable subgroup with the relative property $(\mathrm{T})$ or admits two commuting nonamenable subgroups. In particular the result holds for all free products $\Gamma_{1} * \Gamma_{2}$ where $\Gamma_{1}$ is an infinite property $(\mathrm{T})$ group and $\Gamma_{2} \neq\{e\}$.

Combining the uniqueness of the group measure space Cartan subalgebra with existing orbit equivalence superrigidity results from [Po05, Ki09], we proved in [PV09] several $\mathrm{W}^{*}$-superrigidity theorems: a free ergodic pmp action $\Gamma \curvearrowright(X, \mu)$ is called $\mathrm{W}^{*}$-superrigid if the $\mathrm{II}_{1}$ factor $M=\mathrm{L}^{\infty}(X) \rtimes \Gamma$ entirely remembers the group action. This means that whenever $M=\mathrm{L}^{\infty}(Y) \rtimes \Lambda$, the groups $\Gamma$ and $\Lambda$ are isomorphic and their actions are conjugate.

In FV10 we proved a similar unique group measure space decomposition theorem for all free ergodic pmp actions of certain $\operatorname{HNN}$ extensions $\Gamma=\operatorname{HNN}(H, \Sigma, \theta)$, where $\Sigma$ is amenable and $\Gamma$ satisfies a rigidity assumption as above. In [HPV10] we realized that also certain amalgamated free products $\Gamma=\Gamma_{1} *_{\Sigma} \Gamma_{2}$ over nonamenable groups $\Sigma$ could be covered by the methods of [PV09]. Combined with a theorem of Kida [Ki09] we deduced in [HPV10] that every free ergodic pmp action of the group $\Gamma=\mathrm{SL}(3, \mathbb{Z}) *_{\Sigma} \mathrm{SL}(3, \mathbb{Z})$ is $\mathrm{W}^{*}$-superrigid, when $\Sigma<\mathrm{SL}(3, \mathbb{Z})$ denotes the subgroup of matrices $g$ with $g_{31}=g_{32}=0$.

In the very recent article [CP10, Chifan and Peterson proposed a more conceptual framework to prove the uniqueness of the group measure space decomposition. Their theorem covers all groups $\Gamma$ that admit a nonamenable subgroup with the relative property $(\mathrm{T})$ and that admit an unbounded 1-cocycle into a mixing orthogonal representation. The latter means that there exists an orthogonal representation $\pi: \Gamma \rightarrow \mathcal{O}\left(H_{\mathbb{R}}\right)$ and an unbounded map $b: \Gamma \rightarrow H_{\mathbb{R}}$ satisfying $b(g h)=b(g)+\pi(g) b(h)$ for all $g, h \in \Gamma$, such that for all $\xi, \eta \in H_{\mathbb{R}}$ we have $\langle\pi(g) \xi, \eta\rangle \rightarrow 0$ as $g \rightarrow \infty$. Typical examples arise as free products $\Gamma=\Gamma_{1} * \Gamma_{2}$ containing a nonamenable subgroup with the relative property (T). But Chifan and Peterson cover as well direct products $\Gamma \times \Gamma^{\prime}$ of such groups. This fits perfectly with Monod-Shalom's orbit equivalence rigidity theorems for direct product groups (see [MS02]) and leads to new $\mathrm{W}^{*}$ strong rigidity results.

Amalgamated free products and HNN extensions also admit unbounded 1-cocycles into orthogonal representations $\pi$ through the action on their Bass-Serre tree, but these representations $\pi$ are only mixing relative to the amalgam $\Sigma$. In this paper we generalize Chifan-Peterson's result to groups that admit an unbounded 1-cocycle into an orthogonal representation that is mixing relative to a family of amenable subgroups. As such we obtain a unified treatment for all the uniqueness theorems of the group measure space decomposition in [PV09, FV10, HPV10, CP10].

Although our methods are very close to those in [CP10, we do not use Peterson's technique of unbounded derivations ([Pe06] and [OP08, Section 4]), but rather their dilation into a malleable deformation in the sense of Popa, as proposed by Thomas Sinclair [Si10]. In this way our approach becomes more elementary and we can more directly apply the methods from Popa's deformation/rigidity theory (see [Po06a, Va10] for an overview).

\footnotetext{
${ }^{(3)}$ By definition we call $\Sigma<\Gamma$ weakly malnormal if there exist $g_{1}, \ldots, g_{n} \in \Gamma$ such that $\bigcap_{k=1}^{n} g_{k} \Sigma g_{k}^{-1}$ is finite.
} 


\section{Statements of the main results}

We say that a countable group $\Sigma$ is anti-(T) if there exists a chain of subgroups $\{e\}=\Sigma_{0}<\Sigma_{1}<$ $\cdots<\Sigma_{n}=\Sigma$ such that for all $i=1, \ldots, n$ the subgroup $\Sigma_{i-1}$ is normal in $\Sigma_{i}$ and the quotient $\Sigma_{i} / \Sigma_{i-1}$ has the Haagerup property.

We say that an orthogonal representation $\pi: \Gamma \rightarrow \mathcal{O}\left(H_{\mathbb{R}}\right)$ is mixing relative to a family $\mathcal{S}$ of subgroups of $\Gamma$ if the following holds: for all $\xi, \eta \in H_{\mathbb{R}}$ and $\varepsilon>0$ there exist a finite number of $g_{i}, h_{i} \in \Gamma$ and $\Sigma_{i} \in \mathcal{S}$ such that $|\langle\pi(g) \xi, \eta\rangle|<\varepsilon$ for all $g \in \Gamma-\bigcup_{i=1}^{n} g_{i} \Sigma_{i} h_{i}$.

Definition 1.1. We consider three classes of countable groups $\Gamma$ that admit an unbounded 1cocycle $b: \Gamma \rightarrow H_{\mathbb{R}}$ into an orthogonal representation $\pi: \Gamma \rightarrow \mathcal{O}\left(H_{\mathbb{R}}\right)$ that is mixing relative to a family $\mathcal{S}$ of subgroups of $\Gamma$, with $b$ being bounded on every $\Sigma \in \mathcal{S}$. These three classes correspond to imposing a rigidity on $\Gamma$ versus a softness on the groups in $\mathcal{S}$.

Class $\mathcal{C} . \Gamma$ has a nonamenable subgroup with the relative property $(\mathrm{T})$ and the groups in $\mathcal{S}$ are amenable.

Class $\mathcal{D} . \Gamma$ has an infinite subgroup with the plain property $(\mathrm{T})$ and the groups in $\mathcal{S}$ are anti- $(\mathrm{T})$.

Class $\mathcal{E}$. $\Gamma$ has two commuting nonamenable subgroups, the groups in $\mathcal{S}$ are amenable and $\pi$ is weakly contained in the regular representation.

We also consider the classes $\mathcal{C}_{2}$, respectively $\mathcal{D}_{2}$, consisting of direct products $\Gamma_{1} \times \Gamma_{2}$ where both $\Gamma_{i} \in \mathcal{C}$, respectively both $\Gamma_{i} \in \mathcal{D}$. The groups $\Gamma_{i}$ come with a family $\mathcal{S}_{i}$ of subgroups and we consider the family $\mathcal{S}$ of subgroups of $\Gamma$ of the form $\Sigma_{1} \times \Sigma_{2}, \Sigma_{i} \in \mathcal{S}_{i}$.

The following is our main theorem. Given a group $\Gamma$ in any of the classes introduced above, we 'locate' any possible group measure space Cartan subalgebra $B$ of any crossed product $A \rtimes \Gamma$ and prove that it must have an intertwining bimodule into $A \rtimes \Sigma$ for some $\Sigma \in \mathcal{S}$. We refer to Theorem 2.1 below for the definition of Popa's intertwining bimodules and the corresponding notation $\prec$. If the groups $\Sigma \in \mathcal{S}$ are moreover finite or sufficiently nonnormal, it follows that $\mathrm{L}^{\infty}(X) \rtimes \Gamma$ has a unique group measure space Cartan subalgebra up to unitary conjugacy for all free ergodic pmp actions $\Gamma \curvearrowright(X, \mu)$, see Theorem 1.3. If moreover $\Gamma \curvearrowright(X, \mu)$ is orbit equivalence superrigid, the action $\Gamma \curvearrowright(X, \mu)$ follows $\mathrm{W}^{*}$-superrigid in the sense that the $\mathrm{II}_{1}$ factor $\mathrm{L}^{\infty}(X) \rtimes \Gamma$ entirely remembers the group action that it was constructed from.

Theorem 1.2. Let $\Gamma$ be a group in $\mathcal{C} \cup \mathcal{D} \cup \mathcal{E} \cup \mathcal{C}_{2} \cup \mathcal{D}_{2}$ together with its family $\mathcal{S}$ of subgroups as in Definition 1.1 .

Let $M$ be a $I I_{1}$ factor of the form $M=A \rtimes \Gamma$ where $A$ is of type $I$. Let $p \in M$ be a projection and assume that $p M p=B \rtimes \Lambda$ is another crossed product decomposition with $B$ being of type $I$. Then there exists $\Sigma \in \mathcal{S}$ such that $B \prec A \rtimes \Sigma$.

Theorem 1.2 is sufficiently general to cover all the uniqueness theorems of group measure space Cartan subalgebras from [PV09, FV10, HPV10, CP10]. The precise formulation goes as follows.

We say that $\Sigma \subset \Gamma$ is a weakly malnormal subgroup if there exist $g_{1}, \ldots, g_{n} \in \Gamma$ such that $\bigcap_{k=1}^{n} g_{k} \Sigma g_{k}^{-1}$ is finite. We say that $\Sigma \subset \Gamma$ is relatively malnormal if there exists a subgroup $\Lambda<\Gamma$ of infinite index such that $g \Sigma g^{-1} \cap \Sigma$ is finite for all $g \in \Gamma-\Lambda$.

We consider amalgamated free products $\Gamma_{1} *_{\Sigma} \Gamma_{2}$ and call them nontrivial when $\Gamma_{1} \neq \Sigma \neq \Gamma_{2}$. We also consider $\operatorname{HNN}$ extensions $\operatorname{HNN}(H, \Sigma, \theta)$ w.r.t. a subgroup $\Sigma<H$ and an injective group homomorphism $\theta: \Sigma \rightarrow H$, generated by a copy of $H$ and an extra generator $t$ satisfying $t \sigma t^{-1}=$ $\theta(\sigma)$ for all $\sigma \in \Sigma$. 
Theorem 1.3. For all of the following groups $\Gamma$ and arbitrary free ergodic pmp actions $\Gamma \curvearrowright(X, \mu)$, the $I I_{1}$ factor $M=\mathrm{L}^{\infty}(X) \rtimes \Gamma$ has a unique group measure space Cartan subalgebra up to unitary conjugacy. More generally, if $p\left(\mathrm{M}_{n}(\mathbb{C}) \otimes M\right) p=\mathrm{L}^{\infty}(Y) \rtimes \Lambda$ is an arbitrary group measure space decomposition, there exists $u \in \mathrm{M}_{n}(\mathbb{C}) \otimes M$ such that $p=u^{*} u$, such that $q:=u u^{*}$ belongs to $\mathrm{D}_{n}(\mathbb{C}) \otimes \mathrm{L}^{\infty}(X)$ and such that $u \mathrm{~L}^{\infty}(Y) u^{*}=\left(\mathrm{D}_{n}(\mathbb{C}) \otimes \mathrm{L}^{\infty}(X)\right)$. Here $\mathrm{D}_{n}(\mathbb{C}) \subset \mathrm{M}_{n}(\mathbb{C})$ denotes the subalgebra of diagonal matrices.

1. Any of the groups $\Gamma \in \mathcal{C} \cup \mathcal{D} \cup \mathcal{E} \cup \mathcal{C}_{2} \cup \mathcal{D}_{2}$ such that the family $\mathcal{S}$ consists of relatively malnormal subgroups of $\Gamma$.

2. CP10, Corollary 5.3]. Any of the groups $\Gamma \in \mathcal{C} \cup \mathcal{D} \cup \mathcal{E} \cup \mathcal{C}_{2} \cup \mathcal{D}_{2}$ such that the family $\mathcal{S}$ is reduced to $\{\{e\}\}$, i.e. the case where the orthogonal representations are mixing.

3. [PV09, Theorem 5.2]. $\Gamma$ is a nontrivial amalgamated free product $\Gamma_{1} *_{\Sigma} \Gamma_{2}$ where $\Sigma$ is amenable and weakly malnormal in $\Gamma$ and where $\Gamma$ admits a nonamenable subgroup with the relative property $(T)$ or admits two commuting nonamenable subgroups.

4. [HPV10, Theorem 5]. $\Gamma$ is a nontrivial amalgamated free product $\Gamma_{1} *_{\Sigma} \Gamma_{2}$ where $\Sigma$ is anti-(T) and weakly malnormal in $\Gamma$ and where $\Gamma$ admits an infinite subgroup with property $(T)$.

5. [FV10, Theorem 4.1]. $\Gamma$ is an $H N N$ extension $\operatorname{HNN}(H, \Sigma, \theta)$ such that $\Sigma$ is amenable and weakly malnormal in $\Gamma$ and such that $\Gamma$ admits a nonamenable subgroup with the relative property $(T)$ or admits two commuting nonamenable subgroups.

Ideally Theorem 1.3 could hold for all groups $\Gamma$ in $\mathcal{C} \cup \mathcal{D} \cup \mathcal{E} \cup \mathcal{C}_{2} \cup \mathcal{D}_{2}$ for which $\mathcal{S}$ consists of weakly malnormal subgroups of $\Gamma$, but we were unable to prove such a statement.

As in [OP07, Corollary 4.4] and [PV09, Theorem 1.4] we also get plenty of $\mathrm{II}_{1}$ factors that have no group measure space Cartan subalgebra.

Theorem 1.4. Let $\Gamma$ be any of the groups in Theorem 1.3 and assume moreover that $\Gamma$ has infinite conjugacy classes (icc). Let $\Gamma \curvearrowright(X, \mu)$ be an ergodic pmp action that is not essentially free. Denote $M=\mathrm{L}^{\infty}(X) \rtimes \Gamma$ and observe that $M$ is a $I I_{1}$ factor. This includes the case where $X$ is one point and $M=\mathrm{L} \Gamma$. The $I_{1}$ factors $M^{t}, t>0$, have no group measure space decomposition.

In [Ki10] Kida proves measure equivalence rigidity theorems for amalgamated free product groups $\Gamma$. Such results are complementary to uniqueness theorems for the group measure space Cartan subalgebra since the latter reduce a $\mathrm{W}^{*}$-equivalence to an orbit equivalence to which the measure equivalence rigidity theorems can be applied. It is therefore interesting to notice that Kida does not use the 1-cocycle that goes with the action of $\Gamma$ on the Bass-Serre tree $\mathcal{T}$, but rather the very much related map that associates to three distinct points of $\bar{x}, \bar{y}, \bar{z} \in \partial \mathcal{T}$ the unique vertex of the tree where the three geodesics $\bar{x} \bar{y}, \bar{y} \bar{z}$ and $\bar{z} \bar{x}$ meet.

We have made the choice to write an essentially self-contained article with rather elementary proofs. This means that we provide detailed arguments for a number of lemmas that are well known to some experts. It also gives us the occasion to reprove a number of results, e.g. Peterson's theorem on the solidity of certain group von Neumann algebras, see Theorem 3.6 . 


\section{Structure of the proofs}

Let $M$ be a $\mathrm{II}_{1}$ factor of the form $M=\mathrm{L}^{\infty}(X) \rtimes \Gamma$, where $\Gamma$ is a countable group and $b: \Gamma \rightarrow H_{\mathbb{R}}$ is an unbounded 1-cocycle into an orthogonal representation $\pi: \Gamma \rightarrow \mathcal{O}\left(H_{\mathbb{R}}\right)$. We assume that $\pi$ is mixing relative to a family $\mathcal{S}$ of subgroups of $\Gamma$ and that $b$ is bounded on every $\Sigma \in \mathcal{S}$. Following [BO08, Definition 15.1.1] we call a subset $\mathcal{F} \subset \Gamma$ small relative to $\mathcal{S}$ when $\mathcal{F}$ can be written as a finite union of subsets of the form $g \Sigma h, g, h \in \Gamma, \Sigma \in \mathcal{S}$.

Associated with $(b, \pi)$ is the conditionally negative type function $\psi(g)=\|b(g)\|^{2}$ and the semigroup group $\left(\varphi_{t}\right)_{t>0}$ of completely positive maps

$$
\varphi_{t}: M \rightarrow M: \varphi_{t}\left(a u_{g}\right)=\exp (-t \psi(g)) a u_{g} \quad \text { for all } a \in \mathrm{L}^{\infty}(X), g \in \Gamma .
$$

Assume that $M=\mathrm{L}^{\infty}(Y) \rtimes \Lambda$ is another group measure space decomposition. Also assume that $\Gamma$ satisfies a rigidity assumption, like the presence of a nonamenable subgroup with the (relative) property $(\mathrm{T})$ or the presence of two commuting nonamenable subgroups.

- It is of course impossible to prove that $\Lambda$ automatically inherits similar rigidity properties as $\Gamma$ has. Nevertheless the transfer of rigidity principle from [PV09] allows to prove that there exists a sequence of group elements $s_{n} \in \Lambda$ such that $\varphi_{t} \rightarrow$ id in $\|\cdot\|_{2}$-norm uniformly on $\left\{v_{s_{n}} \mid n \in \mathbb{N}\right\}$. Also the sequence can be taken 'large enough' in the sense that $v_{s_{n}}$ asymptotically leaves all the subspaces spanned by $\left\{a u_{k} \mid a \in \mathrm{L}^{\infty}(X), k \in \mathcal{F}\right\}$ for any fixed subset $\mathcal{F} \subset \Gamma$ that is small relative to $\mathcal{S}$. These matters are dealt with in Proposition 5.1 and Lemma 6.2. The 'larger' the subgroups in $\mathcal{S}$ are allowed to be, the stricter the rigidity assumption on $\Gamma$ must be.

- The unitaries $v_{s_{n}}$ normalize the abelian von Neumann subalgebra $\mathrm{L}^{\infty}(Y)$ and the deformation $\varphi_{t}$ converges to the identity uniformly on the $\left(v_{s_{n}}\right)_{n \in \mathbb{N}}$. In Theorem 4.1 we prove that then $\varphi_{t}$ must converge to the identity uniformly on the unit ball of $\mathrm{L}^{\infty}(Y)$. The roots of this result lie in [Pe09, Theorem 4.1]. Our theorem and its proof are almost identical to [CP10, Theorem 3.2], but we manage to treat as well the case of nonmixing representations.

It is illustrative to compare Theorem 4.1 and its proof to the following group theoretic result inspired by [CTV06. Let $b: \Gamma \rightarrow H_{\mathbb{R}}$ be a 1-cocycle into an orthogonal representation $\pi$ : $\Gamma \rightarrow \mathcal{O}\left(H_{\mathbb{R}}\right)$ that is mixing relative to a family $\mathcal{S}$ of subgroups of $\Gamma$. Assume that $b$ is bounded on every subgroup $\Sigma \in \mathcal{S}$. We say that a sequence $\left(g_{n}\right)$ tends to infinity relative to $\mathcal{S}$ if $\left(g_{n}\right)$ eventually leaves every subset of $\Gamma$ that is small relative to $\mathcal{S}$. Whenever $H<\Gamma$ is an abelian subgroup that is normalized by a sequence $\left(g_{n}\right)$ tending to infinity relative to $\mathcal{S}$ and on which $b$ is bounded, then the 1-cocycle $b$ is bounded on $H$. The proof consists of two cases. Put $\kappa=\sup _{n}\left\|b\left(g_{n}\right)\right\|<\infty$.

Case 1. There is no $h \in H$ such that the sequence $g_{n} h g_{n}^{-1}$ tends to infinity relative to $\mathcal{S}$. We show that $\|b(h)\| \leq 2 \kappa$ for all $h \in H$. To prove this statement, fix $h \in H$. Since $g_{n} h g_{n}^{-1}$ does not tend to infinity relative to $\mathcal{S}$, we can pass to a subsequence and find $\gamma, \gamma^{\prime} \in \Gamma$ and $\Sigma \in \mathcal{S}$ such that $g_{n} h g_{n}^{-1} \in \gamma \Sigma \gamma^{\prime}$ for all $n$. Since $b$ is bounded on $\Sigma$, there exists a vector $\xi \in H_{\mathbb{R}}$ such that $b(\sigma)=\pi(\sigma) \xi-\xi$ for all $\sigma \in \Sigma$. We then find $\xi_{1}, \xi_{2} \in H_{\mathbb{R}}$ such that $b(g)=\pi(g) \xi_{1}+\xi_{2}$ for all $g \in \gamma \Sigma \gamma^{\prime}$. In particular $b\left(g_{n} h g_{n}^{-1}\right)=\pi\left(g_{n} h g_{n}^{-1}\right) \xi_{1}+\xi_{2}$ for all $n$. Since $b\left(g_{n} h g_{n}^{-1}\right)=b\left(g_{n}\right)+\pi\left(g_{n}\right) b(h)-\pi\left(g_{n} h g_{n}^{-1}\right) b\left(g_{n}\right)$ we conclude that

$$
\begin{aligned}
\|b(h)\|^{2} & =\left\langle\pi\left(g_{n}\right) b(h), \pi\left(g_{n}\right) b(h)\right\rangle=\left\langle\pi\left(g_{n}\right) b(h), b\left(g_{n} h g_{n}^{-1}\right)-b\left(g_{n}\right)+\pi\left(g_{n} h g_{n}^{-1}\right) b\left(g_{n}\right)\right\rangle \\
& \leq 2 \kappa\|b(h)\|+\left|\left\langle\pi\left(g_{n}\right) b(h), \pi\left(g_{n} h g_{n}^{-1}\right) \xi_{1}+\xi_{2}\right\rangle\right| \\
& \leq 2 \kappa\|b(h)\|+\left|\left\langle\pi\left(g_{n}\right) \pi(h)^{*} b(h), \xi_{1}\right\rangle\right|+\left|\left\langle\pi\left(g_{n}\right) b(h), \xi_{2}\right\rangle\right| \rightarrow 2 \kappa\|b(h)\|
\end{aligned}
$$


because $\left(g_{n}\right)$ tends to infinity relative to $\mathcal{S}$ and $\pi$ is mixing relative to $\mathcal{S}$. We have shown that $\|b(h)\| \leq 2 \kappa$ for all $h \in H$.

Case 2. There exists $h_{0} \in H$ such that the sequence $h_{n}:=g_{n} h_{0} g_{n}^{-1}$ tends to infinity relative to $\mathcal{S}$. Put $\kappa_{1}=2 \kappa+\left\|b\left(h_{0}\right)\right\|$. Note that $\left\|b\left(h_{n}\right)\right\| \leq \kappa_{1}$ for all $n$. We show that $\|b(h)\| \leq 2 \kappa_{1}$ for all $h \in H$. To prove this statement, fix $h \in H$. Since $H$ is abelian, we have $h=h_{n} h h_{n}^{-1}$ and hence

$$
\begin{aligned}
\|b(h)\|^{2} & =\left\langle b\left(h_{n} h h_{n}^{-1}\right), b(h)\right\rangle=\left\langle b\left(h_{n}\right)+\pi\left(h_{n}\right) b(h)-\pi\left(h_{n} h h_{n}^{-1}\right) b\left(h_{n}\right), b(h)\right\rangle \\
& \leq 2 \kappa_{1}\|b(h)\|+\left|\left\langle\pi\left(h_{n}\right) b(h), b(h)\right\rangle\right| \rightarrow 2 \kappa_{1}\|b(h)\| .
\end{aligned}
$$

We have shown that $\|b(h)\| \leq 2 \kappa_{1}$ for all $h \in H$.

- From the previous step we know that $\varphi_{t} \rightarrow$ id in $\|\cdot\|_{2}$-norm uniformly on the unit ball of $\mathrm{L}^{\infty}(Y)$. We argue by contradiction that there exists a $\Sigma \in \mathcal{S}$ such that $\mathrm{L}^{\infty}(Y) \prec \mathrm{L}^{\infty}(X) \rtimes \Sigma$. If the statement is false Lemma 2.4 provides a sequence of unitaries $b_{n} \in \mathrm{L}^{\infty}(Y)$ such that $b_{n}$ asymptotically leaves all the subspaces spanned by $\left\{a u_{k} \mid a \in \mathrm{L}^{\infty}(X), k \in \mathcal{F}\right\}$ for any fixed subset $\mathcal{F} \subset \Gamma$ that is small relative to $\mathcal{S}$.

By [CP10, Theorem 2.5] -for which we provide a self-contained proof as Theorem 3.10 belowit follows that $\varphi_{t} \rightarrow$ id in $\|\cdot\|_{2}$-norm uniformly on the unit ball of the normalizer of $\mathrm{L}^{\infty}(Y)$, i.e. on the unit ball of the whole of $M$. This is absurd because the 1-cocycle $b$ was assumed to be unbounded.

Also Theorem 3.10 and its proof can be illustrated by a well known group theoretic fact. Let $b: \Gamma \rightarrow H_{\mathbb{R}}$ be a 1-cocycle into an orthogonal representation $\pi: \Gamma \rightarrow \mathcal{O}\left(H_{\mathbb{R}}\right)$ that is mixing relative to a family $\mathcal{S}$ of subgroups of $\Gamma$. Assume that $H<\Gamma$ is a subgroup of $\Gamma$ on which the cocycle is bounded. Denote by $\mathcal{N}_{\Gamma}(H)$ the normalizer of $H$ inside $\Gamma$. If $H$ contains a sequence $\left(h_{n}\right)$ tending to infinity relative to $\mathcal{S}$ (meaning that $\left(h_{n}\right)$ eventually leaves every subset of $\Gamma$ that is small relative to $\mathcal{S}$ ), then $b$ is bounded on $\mathcal{N}_{\Gamma}(H)$. The proof of this fact goes as follows.

Put $\kappa=\sup _{h \in H}\|b(h)\|<\infty$. We show that $\|b(g)\| \leq 2 \kappa$ for all $g \in \mathcal{N}_{\Gamma}(H)$. To prove this statement fix $g \in \mathcal{N}_{\Gamma}(H)$. Write $k_{n}:=g^{-1} h_{n}^{-1} g$ and note that $k_{n} \in H$. By construction $g=h_{n} g k_{n}$. Therefore

$$
\begin{aligned}
\|b(g)\|^{2} & =\langle b(g), b(g)\rangle=\left\langle b\left(h_{n} g k_{n}\right), b(g)\right\rangle=\left\langle b\left(h_{n}\right)+\pi\left(h_{n}\right) b(g)+\pi\left(h_{n} g\right) b\left(k_{n}\right), b(g)\right\rangle \\
& \leq 2 \kappa\|b(g)\|+\left|\left\langle\pi\left(h_{n}\right) b(g), b(g)\right\rangle\right| \rightarrow 2 \kappa\|b(g)\| .
\end{aligned}
$$

It follows that $\|b(g)\| \leq 2 \kappa$ for all $g \in \mathcal{N}_{\Gamma}(H)$.

- Once we know that $\mathrm{L}^{\infty}(Y) \prec \mathrm{L}^{\infty}(X) \rtimes \Sigma$ for some $\Sigma \in \mathcal{S}$, the unitary conjugacy of $\mathrm{L}^{\infty}(X)$ and $\mathrm{L}^{\infty}(X)$ follows from a combination of the regularity of $\mathrm{L}^{\infty}(Y) \subset M$ and a weak malnormality of $\Sigma$ in $\Gamma$, see Lemma 6.3 and [HPV10, Proposition 8].

\section{Acknowledgment}

It is my pleasure to thank Claire Anantharaman, Cyril Houdayer, Jesse Peterson, Sorin Popa and Thomas Sinclair for their helpful comments and careful reading of a first draft of this article.

\section{Popa's intertwining-by-bimodules}

We first recall Popa's intertwining-by-bimodules theorem. 
Theorem 2.1 ([P003, Theorem 2.1 and Corollary 2.3]). Let $(M, \tau)$ be a von Neumann algebra with a faithful normal tracial state $\tau$. Assume that $p \in M$ is a projection and that $P \subset M$ and $B \subset p M p$ are von Neumann subalgebras with $B$ being generated by a group of unitaries $\mathcal{G} \subset \mathcal{U}(B)$. Then the following two statements are equivalent.

- There exists a nonzero partial isometry $v \in \mathrm{M}_{1, n}(\mathbb{C}) \otimes p M$ and a, possibly non-unital, normal $*$-homomorphism $\theta: B \rightarrow \mathrm{M}_{n}(\mathbb{C}) \otimes P$ such that $b v=v \theta(b)$ for all $b \in B$.

- There is no sequence of unitaries $\left(b_{n}\right)$ in $\mathcal{G}$ satisfying $\left\|E_{P}\left(x b_{n} y\right)\right\|_{2} \rightarrow 0$ for all $x, y \in M$.

We write $B \prec P$ if these equivalent conditions hold.

Note that when the von Neumann algebra $M$ is non separable, sequences have to be replaced by nets in the formulation of Theorem 2.1.

Throughout this section we fix a trace preserving action $\Gamma \curvearrowright(N, \tau)$ and denote $M=N \rtimes \Gamma$. We assume that $\mathcal{S}$ is a family of subgroups of $\Gamma$. Following [BO08, Definition 15.1.1] we say that a subset $\mathcal{F} \subset \Gamma$ is small relative to $\mathcal{S}$ if $\mathcal{F}$ can be written as a finite union of subsets of the form $g \Sigma h$, $g, h \in \Gamma, \Sigma \in \mathcal{S}$. Whenever $\mathcal{F} \subset \Gamma$ we denote by $P_{\mathcal{F}}$ the orthogonal projection of $\mathrm{L}^{2}(M)$ onto the closed linear span of $\left\{a u_{g} \mid a \in N, g \in \mathcal{F}\right\}$.

Definition 2.2. Whenever $\mathcal{V} \subset M$ is a norm bounded subset, we write $\mathcal{V} \subset$ approx $N \rtimes \mathcal{S}$ if for every $\varepsilon>0$ there exists a subset $\mathcal{F} \subset \Gamma$ that is small relative to $\mathcal{S}$ such that

$$
\left\|b-P_{\mathcal{F}}(b)\right\|_{2}=\left\|P_{\Gamma-\mathcal{F}}(b)\right\|_{2} \leq \varepsilon \quad \text { for all } b \in \mathcal{V} .
$$

Lemma 2.3. Let $\mathcal{V} \subset M$ be a norm bounded subset satisfying $\mathcal{V} \subset$ approx $N \rtimes \mathcal{S}$. Then for all $x, y \in M$ we have $x \mathcal{V} y \subset_{\text {approx }} N \rtimes \mathcal{S}$.

If $\left(v_{i}\right)$ is a bounded net in $M$ satisfying $\left\|P_{\mathcal{F}}\left(v_{i}\right)\right\|_{2} \rightarrow 0$ for every subset $\mathcal{F} \subset \Gamma$ that is small relative to $\mathcal{S}$, then $\left\|P_{\mathcal{F}}\left(x v_{i} y\right)\right\|_{2} \rightarrow 0$ for every $x, y \in M$ and every subset $\mathcal{F} \subset \Gamma$ that is small relative to $\mathcal{S}$.

Proof. To prove the first statement, by symmetry it suffices to show that $x \mathcal{V} \subset$ approx $N \rtimes \mathcal{S}$. We may assume that $x \in(M)_{1}$ and $\mathcal{V} \subset(M)_{1}$. Choose $\varepsilon>0$. Take a finite subset $\mathcal{F}_{0} \subset \Gamma$ and elements $\left(a_{g}\right)_{g \in \mathcal{F}_{0}}$ of $N$ such that $x_{0}:=\sum_{g \in \mathcal{F}_{0}} a_{g} u_{g}$ satisfies $\left\|x-x_{0}\right\|_{2} \leq \varepsilon / 2$. Put $\kappa=\max \left\{\left\|a_{g}\right\| \mid g \in \mathcal{F}_{0}\right\}$. Take a subset $\mathcal{F} \subset \Gamma$ that is small relative to $\mathcal{S}$ and such that $\left\|P_{\Gamma-\mathcal{F}}(b)\right\|_{2} \leq \varepsilon /\left(2\left|\mathcal{F}_{0}\right| \kappa\right)$ for all $b \in \mathcal{V}$. Define the subset $\mathcal{F}_{1}:=\mathcal{F}_{0} \mathcal{F}$ and note that $\mathcal{F}_{1}$ is small relative to $\mathcal{S}$. We claim that $\left\|P_{\Gamma-\mathcal{F}_{1}}(x b)\right\|_{2} \leq \varepsilon$ for all $b \in \mathcal{V}$. To prove this claim, fix $b \in \mathcal{V}$. Since $\|b\| \leq 1$, we have $\left\|x b-x_{0} b\right\|_{2} \leq\left\|x-x_{0}\right\|_{2} \leq \varepsilon / 2$. So it suffices to prove that $\left\|P_{\Gamma-\mathcal{F}_{1}}\left(x_{0} b\right)\right\|_{2} \leq \varepsilon / 2$. But,

$$
P_{\Gamma-\mathcal{F}_{1}}\left(x_{0} b\right)=\sum_{g \in \mathcal{F}_{0}} a_{g} P_{\Gamma-\mathcal{F}_{1}}\left(u_{g} b\right)
$$

so that

$$
\left\|P_{\Gamma-\mathcal{F}_{1}}\left(x_{0} b\right)\right\|_{2} \leq \sum_{g \in \mathcal{F}_{0}}\left\|a_{g}\right\|\left\|P_{\Gamma-\mathcal{F}_{1}}\left(u_{g} b\right)\right\|_{2} \leq \kappa \sum_{g \in \mathcal{F}_{0}}\left\|P_{\Gamma-\mathcal{F}}(b)\right\|_{2} \leq \frac{\varepsilon}{2} .
$$

This proves the claim and hence also the first statement in the lemma.

To prove the second statement, we can approximate $x$ and $y$ by linear combinations of $a u_{g}, a \in N$, $g \in \Gamma$. Using the Kaplansky density theorem we may assume that $x=u_{g}$ and $y=u_{h}$. But then $P_{\mathcal{F}}\left(u_{g} v_{i} u_{h}\right)=P_{g^{-1} \mathcal{F} h^{-1}}\left(v_{i}\right)$ and we are done. 
The following lemma is essentially contained in [Po03 and Va07, Remark 3.3]. To keep this paper self-contained we provide a short proof. The only reason that nets appear, is because we do not want to make the restriction that the family $\mathcal{S}$ is countable.

Lemma 2.4. Let $p \in M$ be a projection and $B \subset p M p$ a von Neumann subalgebra generated by a group of unitaries $\mathcal{G} \subset \mathcal{U}(B)$. Then the following two statements are equivalent.

- For every $\Sigma \in \mathcal{S}$ we have $B \nprec N \rtimes \Sigma$.

- There exists a net of unitaries $\left(w_{i}\right)$ in $\mathcal{G}$ such that $\left\|P_{\mathcal{F}}\left(w_{i}\right)\right\|_{2} \rightarrow 0$ for every subset $\mathcal{F} \subset \Gamma$ that is small relative to $\mathcal{S}$.

Proof. If $\Sigma \in \mathcal{S}$ and $B \prec N \rtimes \Sigma$, Theorem 2.1 yields a nonzero partial isometry $v \in \mathrm{M}_{1, n}(\mathbb{C}) \otimes p M$ and a normal $*$-homomorphism $\theta: B \rightarrow \mathrm{M}_{n}(\mathbb{C}) \otimes(N \rtimes \Sigma)$ such that $b v=v \theta(b)$ for all $b \in B$. If $\left(w_{i}\right)$ would be a net as in the second statement, Lemma 2.3 implies that $\left\|\left(1 \otimes P_{\mathcal{F}}\right)\left(v^{*} w_{i} v\right)\right\|_{2} \rightarrow 0$ for every subset $\mathcal{F} \subset \Gamma$ that is small relative to $\mathcal{S}$. This holds in particular for $\mathcal{F}=\Sigma$ so that

$$
\left\|\left(\mathrm{id} \otimes E_{N \rtimes \Sigma}\right)\left(v^{*} v\right)\right\|_{2}=\left\|\theta\left(w_{i}\right)\left(\mathrm{id} \otimes E_{N \rtimes \Sigma}\right)\left(v^{*} v\right)\right\|_{2}=\left\|\left(\mathrm{id} \otimes E_{N \rtimes \Sigma}\right)\left(v^{*} w_{i} v\right)\right\|_{2} \rightarrow 0 .
$$

We arrive at the contradiction that $v=0$.

Conversely assume that for every $\Sigma \in \mathcal{S}$ we have $B \nprec N \rtimes \Sigma$. Let $\mathcal{F} \subset \Gamma$ be a subset that is small relative to $\mathcal{S}$ and let $\varepsilon>0$. We have to prove the existence of $w \in \mathcal{G}$ such that $\left\|P_{\mathcal{F}}(w)\right\|_{2}<\varepsilon$. Write $\mathcal{F}=\bigcup_{k=1}^{n} g_{k} \Sigma_{k} h_{k}$ with $\Sigma_{k} \in \mathcal{S}$ and $g_{k}, h_{k} \in \Gamma$. Consider in $\mathrm{M}_{n}(\mathbb{C}) \otimes M$ the diagonal subalgebra $P:=\bigoplus_{k} N \rtimes \Sigma_{k}$. Since for every $k=1, \ldots, n$ we have $B \nprec N \rtimes \Sigma_{k}$, the first criterion of Theorem 2.1 implies that $B \nprec P$. Then the second criterion in Theorem 2.1 provides a sequence of unitaries $w_{i} \in \mathcal{G}$ such that

$$
\left\|E_{N \rtimes \Sigma_{k}}\left(x w_{i} y\right)\right\|_{2} \rightarrow 0 \text { for all } x, y \in M, k=1, \ldots, n .
$$

Applying this to $x=u_{g_{k}}^{*}$ and $y=u_{h_{k}}^{*}$, this means that $\left\|P_{g_{k} \Sigma_{k} h_{k}}\left(w_{i}\right)\right\|_{2} \rightarrow 0$. Hence $\left\|P_{\mathcal{F}}\left(w_{i}\right)\right\|_{2} \rightarrow 0$ and it suffices to take $w=w_{i}$ for $i$ sufficiently large.

The following lemma clarifies the relation between the approximate containment $\subset_{\text {approx }}$ and the intertwining relation $\prec$.

Lemma 2.5. Let $p \in M$ be a projection and $B \subset p M p$ a von Neumann subalgebra. The following two statements are equivalent.

1. There exists a $\Sigma \in \mathcal{S}$ such that $B \prec N \rtimes \Sigma$.

2. There exists a nonzero projection $q \in B^{\prime} \cap p M p$ such that $(B q)_{1} \subset_{\text {approx }} N \rtimes \mathcal{S}$.

Also the following two statements are equivalent.

a. For every nonzero projection $q \in B^{\prime} \cap p M p$ there exists a $\Sigma \in \mathcal{S}$ such that $B q \prec N \rtimes \Sigma$.

b. We have $(B)_{1} \subset$ approx $N \rtimes \mathcal{S}$. 
Proof. 1 $\Rightarrow 2$, Take $\Sigma \in \mathcal{S}$ such that $B \prec N \rtimes \Sigma$. Theorem 2.1 provides a nonzero partial isometry $v \in \mathrm{M}_{1, n}(\mathbb{C}) \otimes p M$ such that $q:=v v^{*}$ belongs to $B^{\prime} \cap p M p$ and satisfies $B q \subset v\left(\mathrm{M}_{n}(\mathbb{C}) \otimes(N \rtimes \Sigma)\right) v^{*}$. It follows from Lemma 2.3 that $(B q)_{1} \subset$ approx $N \rtimes \mathcal{S}$.

$\neg 1 \Rightarrow \neg 2$. We assume that for all $\Sigma \in \mathcal{S}$ we have $B \nprec N \rtimes \Sigma$. Then Lemma 2.4 provides a net of unitaries $\left(w_{i}\right)$ in $\mathcal{U}(B)$ such that $\left\|P_{\mathcal{F}}\left(w_{i}\right)\right\|_{2} \rightarrow 0$ for every subset $\mathcal{F} \subset \Gamma$ that is small relative to $\mathcal{S}$. Take a nonzero projection $q \in B^{\prime} \cap p M p$. We conclude from Lemma 2.3 that $\left\|P_{\mathcal{F}}\left(w_{i} q\right)\right\|_{2} \rightarrow 0$ for every subset $\mathcal{F} \subset \Gamma$ that is small relative to $\mathcal{S}$. Since $w_{i} q \in(B q)_{1}$ and $\left\|w_{i} q\right\|_{2}=\|q\|_{2}>0$ for all $i$, we see that $(B q)_{1}$ is not approximately contained in $N \rtimes \mathcal{S}$.

$\mathrm{a} \Rightarrow \mathrm{b}$. By the assumption in statement $\mathrm{a}$ and using the already proven implication $1 \Rightarrow 2$, we can take an orthogonal family of projections $q_{i} \in B^{\prime} \cap p M p$ such that $\sum_{i} q_{i}=p$ and $\left(B q_{i}\right)_{1} \subset_{\text {approx }} N \rtimes \mathcal{S}$ for all $i$. It follows that $(B)_{1} \subset_{\text {approx }} N \rtimes \mathcal{S}$.

$\mathrm{b} \Rightarrow$ a. Assume that $(B)_{1} \subset_{\text {approx }} N \rtimes \mathcal{S}$ and let $q \in B^{\prime} \cap p M p$ be a nonzero projection. We conclude from Lemma 2.3 that $(B q)_{1} \subset$ approx $N \rtimes \mathcal{S}$. The already proven implication $2 \Rightarrow 1$ implies that there exists a $\Sigma \in \mathcal{S}$ such that $B q \prec N \rtimes \Sigma$.

Whenever $p \in M$ is a projection and $B \subset p M p$ is a von Neumann subalgebra we find as follows a unique projection $q \in B^{\prime} \cap p M p$ such that $(B q)_{1} \subset_{\text {approx }} N \rtimes \mathcal{S}$ and such that $B(p-q) \nprec N \rtimes \Sigma$ for all $\Sigma \in \mathcal{S}$.

Proposition 2.6. Let $p \in M$ be a projection and $B \subset p M p$ a von Neumann subalgebra generated by a group of unitaries $\mathcal{G} \subset \mathcal{U}(B)$. Denote by $Q$ the normalizer of $B$ inside $p M p$. The set of projections

$$
\mathcal{P}:=\left\{q_{0} \mid q_{0} \text { is a projection in } B^{\prime} \cap p M p \text { and }\left(B q_{0}\right)_{1} \subset_{\text {approx }} N \rtimes \mathcal{S}\right\}
$$

attains its maximum in a unique projection $q \in \mathcal{P}$. This projection $q$ belongs to $\mathcal{Z}(Q)$.

Moreover there exists a net of unitaries $\left(w_{i}\right)$ in $\mathcal{G}$ such that $\left\|P_{\mathcal{F}}\left(w_{i}(p-q)\right)\right\|_{2} \rightarrow 0$ for every subset $\mathcal{F} \subset \Gamma$ that is small relative to $\mathcal{S}$.

Proof. Let $q_{k} \in B^{\prime} \cap p M p$ be a maximal orthonormal sequence of nonzero projections in $\mathcal{P}$. Define $q=\sum_{k} q_{k}$. It is easy to prove that $q \in \mathcal{P}$. We prove that $q$ is the maximum of $\mathcal{P}$. Assume that $q^{\prime} \in \mathcal{P}$ and that $q^{\prime} \not \leq q$. It follows that $T:=(p-q) q^{\prime}(p-q)$ is a nonzero operator in $B^{\prime} \cap p M p$. Take $S \in B^{\prime} \cap p M p$ such that $T S$ is a nonzero spectral projection $q^{\prime \prime}$ of $T$. Note that the projection $q^{\prime \prime}$ is orthogonal to $q$ and that the formula

$$
(B)_{1} q^{\prime \prime}=(p-q)(B)_{1} q^{\prime}(p-q) S,
$$

together with Lemma 2.3 implies that $\left(B q^{\prime \prime}\right)_{1} \subset_{\text {approx }} N \rtimes \mathcal{S}$. This contradicts the maximality of the sequence $\left(q_{k}\right)$. So, $\mathcal{P}$ attains its maximum in $q \in \mathcal{P}$.

If $u \in \mathcal{N}_{p M p}(B)$, the fact that $(B)_{1} q \subset$ approx $N \rtimes \mathcal{S}$ and Lemma 2.3 imply that $u(B)_{1} q u^{*} \subset_{\text {approx }}$ $N \rtimes \mathcal{S}$. But $u(B)_{1} q u^{*}=(B)_{1} u q u^{*}$ and it follows that $u q u^{*} \leq q$. Hence, $q$ commutes with $Q$. In particular, $q$ commutes with $B$ so that $q \in Q$. Hence, $q \in \mathcal{Z}(Q)$.

By Lemma 2.4 it remains to prove that for all $\Sigma \in \mathcal{S}$ we have $B(p-q) \nprec N \rtimes \Sigma$. If the contrary would be true, the implication $1 \Rightarrow 2$ in Lemma 2.5 provides a nonzero projection $q^{\prime} \in B^{\prime} \cap p M p$ such that $q^{\prime} \leq p-q$ and such that $\left(B q^{\prime}\right)_{1} \subset_{\text {approx }} N \rtimes \mathcal{S}$. This contradicts the maximality of $q$. 
Lemma 2.7. Assume that $\mathcal{S}_{1}$ and $\mathcal{S}_{2}$ are two families of subgroups of $\Gamma$. Define the family $\mathcal{S}$ consisting of the subgroups $\Sigma_{1} \cap g \Sigma_{2} g^{-1}, \Sigma_{i} \in \mathcal{S}_{i}, g \in \Gamma$. Let $B \subset p M p$ be a von Neumann subalgebra. If $(B)_{1} \subset$ approx $N \rtimes \mathcal{S}_{i}$ for $i=1,2$, then $(B)_{1} \subset$ approx $N \rtimes \mathcal{S}$.

Proof. It suffices to make the following observation: if $\mathcal{F}_{i} \subset \Gamma$ are small relative to $\mathcal{S}_{i}$ for $i=1,2$, then $\mathcal{F}_{1} \cap \mathcal{F}_{2}$ is small relative to $\mathcal{S}$ and $P_{\mathcal{F}_{1} \cap \mathcal{F}_{2}}=P_{\mathcal{F}_{1}} \circ P_{\mathcal{F}_{2}}$.

\section{Malleable deformations coming from group 1-cocycles}

\subsection{Malleable deformation by a one-parameter group of automorphisms}

Throughout this section $\pi: \Gamma \rightarrow \mathcal{O}\left(H_{\mathbb{R}}\right)$ is an orthogonal representation of a countable group $\Gamma$ and $b: \Gamma \rightarrow H_{\mathbb{R}}$ is a 1 -cocycle, i.e. a map satisfying

$$
b(g h)=b(g)+\pi(g) b(h) \quad \text { for all } g, h \in \Gamma .
$$

The function $\Gamma \rightarrow \mathbb{C}: g \mapsto\|b(g)\|^{2}$ is conditionally of negative type. Whenever $\Gamma \curvearrowright(N, \tau)$ is a trace preserving action and $M=N \rtimes \Gamma$, we get a semigroup $\left(\varphi_{t}\right)_{t>0}$ of unital tracial completely positive maps

$$
\varphi_{t}: M \rightarrow M: \varphi_{t}\left(a u_{g}\right)=\exp \left(-t\|b(g)\|^{2}\right) a u_{g} \quad \text { for all } a \in N, g \in \Gamma .
$$

Following [Si10, beginning of Section 3] we construct a malleable deformation of $M$ in the sense of Popa (see [Po06a, Section 6]), i.e. a canonical larger finite von Neumann algebra $M \subset \widetilde{M}$ together with a 1-parameter group of automorphisms $\left(\alpha_{t}\right)_{t \in \mathbb{R}}$ of $\widetilde{M}$ such that $\varphi_{t^{2}}(x)=E_{M}\left(\alpha_{t}(x)\right)$ for all $x \in M$ and $t \in \mathbb{R}$.

Denote by $\Gamma \curvearrowright(Z, \eta)$ the Gaussian action associated with $\pi$. Put $D=\mathrm{L}^{\infty}(Z)$ and denote by $\tau$ the trace on $D$ given by integration with respect to $\eta$. Denote by $\left(\sigma_{g}\right)_{g \in \Gamma}$ the Gaussian action viewed as an action by trace preserving automorphisms of $D$. For our purposes it is most convenient to consider $(D, \tau)$ as the unique pair of a von Neumann algebra $D$ with a faithful tracial state $\tau$ such that $D$ is generated by unitary elements $\omega(\xi), \xi \in H_{\mathbb{R}}$, satisfying

- $\omega(0)=1, \omega\left(\xi_{1}+\xi_{2}\right)=\omega\left(\xi_{1}\right) \omega\left(\xi_{2}\right)$ for all $\xi_{1}, \xi_{2} \in H_{\mathbb{R}}$ and $\omega(\xi)^{*}=\omega(-\xi)$ for all $\xi \in H_{\mathbb{R}}$,

- $\tau(\omega(\xi))=\exp \left(-\|\xi\|^{2}\right)$ for all $\xi \in H_{\mathbb{R}}$.

Moreover, $\sigma_{g}(\omega(\xi))=\omega(\pi(g) \xi)$. Note that the linear span of all $\omega(\xi), \xi \in H_{\mathbb{R}}$, is a dense $*-$ subalgebra of $D$.

Define $\widetilde{M}=(D \bar{\otimes} N) \rtimes \Gamma$ where $\Gamma \curvearrowright D \bar{\otimes} N$ is the diagonal action. The formula

$$
\alpha_{t}(x)=x \text { for all } x \in D \bar{\otimes} N \text { and } \alpha_{t}\left(u_{g}\right)=(\omega(t b(g)) \otimes 1) u_{g} \text { for all } g \in \Gamma
$$

provides the required 1-parameter group of automorphisms.

We finally prove that the deformation $\left(\alpha_{t}\right)_{t \in \mathbb{R}}$ is $s$-malleable in the sense of [Po06a, Section 6]. The formula $\beta(\omega(\xi))=\omega(-\xi)=\omega(\xi)^{*}$ for all $\xi \in H_{\mathbb{R}}$, defines a trace preserving automorphism of $D$. This automorphism extends to an automorphism $\beta$ of $\widetilde{M}$ satisfying $\beta(x)=x$ for all $x \in M$. One easily checks that $\beta$ satisfies the $s$-malleability conditions : $\beta^{2}=$ id and $\beta \circ \alpha_{t}=\alpha_{-t} \circ \beta$ for all $t \in \mathbb{R}$. 


\subsection{Two easy inequalities}

We start by proving a version of the 'transversality inequality' from [Po06c, Lemma 2.1].

Lemma 3.1. Denote for $x \in M, \delta_{t}(x)=\alpha_{t}(x)-E_{M}\left(\alpha_{t}(x)\right)$. Then,

$$
\left\|\delta_{t}(x)\right\|_{2} \leq\left\|x-\alpha_{t}(x)\right\|_{2} \leq \sqrt{2}\left\|\delta_{t}(x)\right\|_{2} \quad \text { for all } x \in M, t \in \mathbb{R} .
$$

So, studying convergence of $\alpha_{t} \rightarrow \mathrm{id}$ is equivalent to studying convergence of $\delta_{t} \rightarrow 0$.

Also, if $x \in M$ and $|t|$ decreases, the expressions $\left\|\delta_{t}(x)\right\|_{2}$ and $\left\|x-\alpha_{t}(x)\right\|_{2}$ decrease.

Proof. Define $f_{t}(g)=\exp \left(-t^{2}\|b(g)\|^{2}\right)$ and note that $0<f_{t}(g) \leq 1$ for all $g \in \Gamma$ and $t \in \mathbb{R}$. Take $x \in M$ and write $x=\sum_{g \in \Gamma} x_{g} u_{g}$ with $x_{g} \in N$. It is easy to check that

$$
\left\|\delta_{t}(x)\right\|_{2}^{2}=\sum_{g \in \Gamma}\left(1-f_{t}(g)^{2}\right)\left\|x_{g}\right\|_{2}^{2} \quad \text { and } \quad\left\|x-\alpha_{t}(x)\right\|_{2}^{2}=2 \sum_{g \in \Gamma}\left(1-f_{t}(g)\right)\left\|x_{g}\right\|_{2}^{2} .
$$

Using the inequalities $1-f_{t}(g)^{2} \leq 2\left(1-f_{t}(g)\right) \leq 2\left(1-f_{t}(g)^{2}\right)$, the lemma follows.

Lemma 3.2. For all $x, y \in(\widetilde{M})_{1}$ and all $t, \varepsilon>0$, there exists $s>0$ such that

$$
\left\|E_{M}\left(x \alpha_{t}(a) y\right)\right\|_{2} \leq\left\|E_{M}\left(\alpha_{s}(a)\right)\right\|_{2}+\varepsilon \quad \text { for all } a \in(M)_{1} .
$$

Proof. Fix $x, y \in(\widetilde{M})_{1}$ and fix $t, \varepsilon>0$. By the Kaplansky density theorem choose $x_{1}, \ldots, x_{n}$, $y_{1}, \ldots, y_{m} \in M$ and $\xi_{1}, \ldots, \xi_{n}, \eta_{1}, \ldots, \eta_{m} \in H_{\mathbb{R}}$ such that the elements

$$
x_{0}:=\sum_{i=1}^{n} x_{i} \omega\left(\xi_{i}\right) \quad \text { and } \quad y_{0}:=\sum_{j=1}^{m} \omega\left(\eta_{j}\right) y_{j}
$$

satisfy $\left\|x_{0}\right\|,\left\|y_{0}\right\| \leq 1$ and $\left\|x-x_{0}\right\|_{2} \leq \varepsilon / 4,\left\|y-y_{0}\right\|_{2} \leq \varepsilon / 4$.

Define $\kappa_{1}=\max \left\{\left\|x_{i}\right\| \mid i=1, \ldots, n\right\}$ and $\kappa_{2}=\max \left\{\left\|y_{j}\right\| \mid j=1, \ldots, m\right\}$. Also put $\gamma_{1}=$ $\max \left\{\left\|\xi_{i}\right\| \mid i=1, \ldots, n\right\}$ and $\gamma_{2}=\max \left\{\left\|\eta_{j}\right\| \mid j=1, \ldots, m\right\}$. Choose $\rho>0$ large enough such that $\exp \left(-\rho^{2}\right) \leq \varepsilon /\left(4 n \kappa_{1} m \kappa_{2}\right)$. Then put $\kappa=\left(\rho+\gamma_{1}+\gamma_{2}\right) / t$. Finally take $s>0$ small enough such that $\exp \left(-2 s^{2} \kappa^{2}\right) \geq 1-(\varepsilon / 4)^{2}$. We claim that this $s$ satisfies the conclusions of the lemma.

To prove the claim define $\mathcal{F}:=\{g \in \Gamma \mid\|b(g)\| \leq \kappa\}$. Whenever $g \in \Gamma-\mathcal{F}$, we have $\|t b(g)\| \geq$ $\rho+\gamma_{1}+\gamma_{2}$ and hence $\left\|\xi_{i}+t b(g)+\pi(g) \eta_{j}\right\| \geq \rho$ so that

$$
\exp \left(-\left\|\xi_{i}+t b(g)+\pi(g) \eta_{j}\right\|^{2}\right) \leq \exp \left(-\rho^{2}\right) \leq \frac{\varepsilon}{4 n \kappa_{1} m \kappa_{2}} \quad \text { for all } i=1, \ldots, n, j=1, \ldots, m .
$$

For any subset $\mathcal{G} \subset \Gamma$ we denote by $P_{\mathcal{G}}$ the orthogonal projection of $\mathrm{L}^{2}(M)$ onto the closed linear span of $\left\{a u_{g} \mid a \in N, g \in \mathcal{G}\right\}$. We decompose every $a \in M$ in its Fourier expansion $a=\sum_{g \in \Gamma} a_{g} u_{g}$ with $a_{g} \in N$. Noticing that for all $a \in M$ we have

$$
E_{M}\left(\omega\left(\xi_{i}\right) \alpha_{t}\left(P_{\Gamma-\mathcal{F}}(a)\right) \omega\left(\eta_{j}\right)\right)=\sum_{g \in \Gamma-\mathcal{F}} \exp \left(-\left\|\xi_{i}+t b(g)+\pi(g) \eta_{j}\right\|^{2}\right) a_{g} u_{g},
$$

it follows that for all $a \in(M)_{1}$ we have

$$
\left\|E_{M}\left(\omega\left(\xi_{i}\right) \alpha_{t}\left(P_{\Gamma-\mathcal{F}}(a)\right) \omega\left(\eta_{j}\right)\right)\right\|_{2} \leq \frac{\varepsilon}{4 n \kappa_{1} m \kappa_{2}} .
$$


Multiplying with $x_{i}$ on the left and with $y_{j}$ on the right and summing over $i$ and $j$, we conclude that

$$
\left\|E_{M}\left(x_{0} \alpha_{t}\left(P_{\Gamma-\mathcal{F}}(a)\right) y_{0}\right)\right\|_{2} \leq \frac{\varepsilon}{4}
$$

for all $a \in(M)_{1}$. Since $\left\|x_{0}\right\|,\left\|y_{0}\right\| \leq 1$, it follows that for all $a \in(M)_{1}$ we have

$$
\left\|E_{M}\left(x_{0} \alpha_{t}(a) y_{0}\right)\right\|_{2} \leq\left\|P_{\mathcal{F}}(a)\right\|_{2}+\frac{\varepsilon}{4} .
$$

Whenever $g \in \mathcal{F}$ we have $\|b(g)\| \leq \kappa$ and therefore $\exp \left(-2\|s b(g)\|^{2}\right) \geq 1-(\varepsilon / 4)^{2}$. So, for all $a \in(M)_{1}$ we get

$$
\begin{aligned}
\left\|P_{\mathcal{F}}(a)\right\|_{2}^{2} & =\sum_{g \in \mathcal{F}}\left\|a_{g}\right\|_{2}^{2} \leq \sum_{g \in \Gamma}\left(\exp \left(-2\|s b(g)\|^{2}\right)+\frac{\varepsilon^{2}}{16}\right)\left\|a_{g}\right\|_{2}^{2} \\
& =\left\|E_{M}\left(\alpha_{s}(a)\right)\right\|_{2}^{2}+\left(\frac{\varepsilon}{4}\|a\|_{2}\right)^{2} \leq\left(\left\|E_{M}\left(\alpha_{s}(a)\right)\right\|_{2}+\frac{\varepsilon}{4}\right)^{2} .
\end{aligned}
$$

Altogether it follows that

$$
\left\|E_{M}\left(x_{0} \alpha_{t}(a) y_{0}\right)\right\|_{2} \leq\left\|E_{M}\left(\alpha_{s}(a)\right)\right\|_{2}+\frac{\varepsilon}{2}
$$

for all $a \in(M)_{1}$. Our choice of $x_{0}$ and $y_{0}$ then imply the claim.

\subsection{The maximal projection under which the malleable deformation is uniform}

Lemma 3.3. Let $p \in M$ be a projection and $B \subset p M p$ a von Neumann subalgebra. Define $Q \subset p M p$ as the normalizer of $B$ inside $p M p$. The set of projections

$$
\mathcal{P}:=\left\{q_{0} \mid q_{0} \text { is a projection in } B^{\prime} \cap p M p \text { and } \alpha_{t} \rightarrow \text { id in }\|\cdot\|_{2} \text {-norm uniformly on }\left(B q_{0}\right)_{1}\right\}
$$

attains its maximum in a unique projection $q \in \mathcal{P}$. This projection $q$ belongs to $\mathcal{Z}(Q)$.

Proof. Let $q_{k} \in B^{\prime} \cap p M p$ be a maximal orthogonal sequence of nonzero elements of $\mathcal{P}$. Put $q=\sum_{k} q_{k}$. It is easy to check that $q \in \mathcal{P}$. We claim that $q$ is the maximum of $\mathcal{P}$. So assume that $e \in \mathcal{P}$. We have to prove that $e \leq q$. If this is not the case, $T:=(p-q) e(p-q)$ is nonzero. Since $\alpha_{t} \rightarrow$ id uniformly on $(B)_{1} e$, the same is true on

$$
(B)_{1} T=(p-q)(B)_{1} e(p-q) .
$$

Since $T$ is nonzero we can find a bounded operator $S \in B^{\prime} \cap p M p$ such that $T S$ is a nonzero spectral projection $f$ of $T$. It follows that $\alpha_{t} \rightarrow$ id uniformly on $(B)_{1} f=(B)_{1} T S$. This contradicts the maximality of the sequence $\left(q_{k}\right)$.

We finally prove that $q \in \mathcal{Z}(Q)$. Let $u \in \mathcal{N}_{p M p}(B)$. Since $\alpha_{t} \rightarrow$ id uniformly on $(B)_{1} q$, the same is true on $u(B)_{1} q u^{*}$, which equals $(B)_{1} u q u^{*}$. Hence $u q u^{*} \leq q$ which means that $u q u^{*}=q$ for all $u \in \mathcal{N}_{p M p}(B)$. So, $q \in Q^{\prime} \cap p M p=\mathcal{Z}(Q)$.

Assume that $B \subset p M p$ is a von Neumann subalgebra and let $q \in B^{\prime} \cap p M p$ be as in the previous lemma, the largest projection such that $\alpha_{t} \rightarrow$ id uniformly on $(B)_{1} q$. In good cases, it follows that on $(B)_{1}(p-q)$ the convergence is the furthest possible from being uniform, in the sense that we can find a sequence of unitaries $\left(w_{n}\right)$ in $\mathcal{U}(B)$ such that for every $t>0$ we have that 
$\left\|E_{M}\left(\alpha_{t}\left(w_{n}(p-q)\right)\right)\right\|_{2} \rightarrow 0$ as $n \rightarrow \infty$. These good cases correspond to $\pi$ being mixing relative to a family $\mathcal{S}$ of subgroups of $\Gamma$ such that the 1-cocycle $b$ is bounded on every $\Sigma \in \mathcal{S}$, see Proposition 3.9 below.

To establish uniform convergence of $\alpha_{t}$ on $B q$, it often suffices to prove uniform convergence on $r \mathcal{G} r$ where $r \leq q$ is a smaller projection and $\mathcal{G}$ is a group of unitaries generating $B$. The precise result goes as follows.

Proposition 3.4. Let $p \in M$ be a projection and $B \subset p M p$ a von Neumann subalgebra generated by a group of unitaries $\mathcal{G} \subset \mathcal{U}(B)$. Let $r \in p M p$ be any projection and assume that $\alpha_{t} \rightarrow \mathrm{id}$ in $\|\cdot\|_{2}$-norm uniformly on the set $r \mathcal{G} r$.

Define $Q$ as the normalizer of $B$ inside $p M p$ and denote by $q$ the smallest projection in $\mathcal{Z}(Q)$ satisfying $r \leq q$. Then, $\alpha_{t} \rightarrow$ id in $\|\cdot\|_{2}$-norm uniformly on the unit ball of $B q$.

Proof. For $x \in M$ we write $\delta_{t}(x)=\alpha_{t}(x)-E_{M}\left(\alpha_{t}(x)\right)$. By Lemma 3.1 we have that $\delta_{t} \rightarrow 0$ in $\|\cdot\|_{2}$-norm uniformly on $r \mathcal{G} r$. Define $T:=E_{B^{\prime} \cap p M p}(r)$. We claim that $\delta_{t} \rightarrow 0$ in $\|\cdot\|_{2}$-norm uniformly on $T \mathcal{G} T$. To prove this claim, choose $\varepsilon>0$. Note that $T$ coincides with the unique element of minimal $\|\cdot\|_{2}$-norm in the $\|\cdot\|_{2^{-}}$closed convex hull of $\left\{u r u^{*} \mid u \in \mathcal{G}\right\}$. So we can take $u_{1}, \ldots, u_{n} \in \mathcal{G}$ and $\lambda_{1}, \ldots, \lambda_{n} \in[0,1]$ such that $\sum_{i} \lambda_{i}=1$ and such that

$$
T_{0}:=\sum_{i=1}^{n} \lambda_{i} u_{i} r u_{i}^{*}
$$

satisfies $\left\|T-T_{0}\right\|_{2} \leq \varepsilon$. Note that $\left\|T_{0}\right\| \leq 1$.

Choose $t>0$ small enough such that $\| \delta_{t}($ rur $) \|_{2} \leq \varepsilon$ for all $u \in \mathcal{G}$ and such that $\left\|\alpha_{t}\left(u_{i}\right)-u_{i}\right\|_{2} \leq \varepsilon$ for all $i=1, \ldots, n$. The latter implies that $\left\|\delta_{t}\left(u_{i} x u_{j}^{*}\right)-u_{i} \delta_{t}(x) u_{j}^{*}\right\|_{2} \leq 4 \varepsilon$ for all $x \in(M)_{1}$. Whenever $u \in \mathcal{G}$, we have $u_{i}^{*} u u_{j} \in \mathcal{G}$ and hence

$$
\left\|\delta_{t}\left(u_{i} r u_{i}^{*} u u_{j} r u_{j}^{*}\right)\right\|_{2} \leq\left\|u_{i} \delta_{t}\left(r u_{i}^{*} u u_{j} r\right) u_{j}^{*}\right\|_{2}+4 \varepsilon \leq 5 \varepsilon .
$$

Taking convex combinations it follows that $\left\|\delta_{t}\left(T_{0} u T_{0}\right)\right\|_{2} \leq 5 \varepsilon$ for all $u \in \mathcal{G}$. Since $\left\|T-T_{0}\right\|_{2} \leq \varepsilon$ and both $\|T\|,\left\|T_{0}\right\| \leq 1$, it follows that $\left\|\delta_{t}(T u T)\right\|_{2} \leq 7 \varepsilon$ for all $u \in \mathcal{G}$, hence proving the claim.

Since $T$ commutes with the elements in $\mathcal{G}$ and since for all $u \in \mathcal{G}$ we have $\left\|\delta_{t}\left(u T^{2}\right)-\delta_{t}(u) T^{2}\right\|_{2} \leq$ $2\left\|\alpha_{t}\left(T^{2}\right)-T^{2}\right\|_{2}$, it follows that $\left\|\delta_{t}(u) T^{2}\right\|_{2} \rightarrow 0$ uniformly in $u \in \mathcal{G}$. For every $\delta>0$ define the spectral projection $q_{\delta}=\chi_{(\delta,+\infty)}\left(T^{2}\right)$ and take bounded operators $T_{\delta} \in B^{\prime} \cap p M p$ such that $T^{2} T_{\delta}=q_{\delta}$. It follows that for every $\delta>0$, we have that $\left\|\delta_{t}(u) q_{\delta}\right\|_{2} \rightarrow 0$ uniformly in $u \in \mathcal{G}$. Denote by $q_{0}$ the support projection of $T$. When $\delta \rightarrow 0$ we have $\left\|q_{0}-q_{\delta}\right\|_{2} \rightarrow 0$. Hence, $\left\|\delta_{t}(u) q_{0}\right\|_{2} \rightarrow 0$ uniformly in $u \in \mathcal{G}$. So, $\alpha_{t} \rightarrow$ id in $\|\cdot\|_{2}$-norm uniformly on $\mathcal{G} q_{0}$.

Note that $r \leq q_{0}$ and that actually $q_{0}$ precisely is the smallest projection in $B^{\prime} \cap p M p$ satisfying $r \leq q_{0}$. By Lemma 3.3 it remains to prove that $\alpha_{t} \rightarrow$ id in $\|\cdot\|_{2}$-norm uniformly on the unit ball of $B q_{0}$. Choose $\varepsilon>0$. Take $t>0$ such that $\left\|u q_{0}-\alpha_{t}\left(u q_{0}\right)\right\|_{2} \leq \varepsilon$ for all $u \in \mathcal{G}$. In particular $\left\|q_{0}-\alpha_{t}\left(q_{0}\right)\right\|_{2} \leq \varepsilon$. Define $v \in \widetilde{M}$ as the element of smallest $\|\cdot\|_{2}$-norm in the $\|\cdot\|_{2}$-closed convex hull of $\left\{u q_{0} \alpha_{t}\left(q_{0} u^{*}\right) \mid u \in \mathcal{G}\right\}$. Note that $b v=v \alpha_{t}(b)$ for all $b \in B q_{0}$ and that $\left\|v-q_{0}\right\|_{2} \leq \varepsilon$. Whenever $b \in(B)_{1} q_{0}$ we have

$$
\left\|\alpha_{t}(b)-v \alpha_{t}(b)\right\|_{2}=\left\|\left(\alpha_{t}\left(q_{0}\right)-v\right) \alpha_{t}(b)\right\|_{2} \leq\left\|\alpha_{t}\left(q_{0}\right)-v\right\|_{2} \leq\left\|\alpha_{t}\left(q_{0}\right)-q_{0}\right\|_{2}+\left\|q_{0}-v\right\|_{2} \leq 2 \varepsilon .
$$

We also have for all $b \in(B)_{1} q_{0}$ that $\|b v-b\|_{2} \leq\left\|b\left(v-q_{0}\right)\right\|_{2} \leq \varepsilon$. Since $b v=v \alpha_{t}(b)$ we conclude that $\left\|\alpha_{t}(b)-b\right\|_{2} \leq 3 \varepsilon$ for all $b \in(B)_{1} q_{0}$. This ends the proof of the proposition. 


\subsection{Cocycles into representations that are weakly contained in the regular rep- resentation}

Lemma 3.5. Assume that $\pi: \Gamma \rightarrow \mathcal{O}\left(H_{\mathbb{R}}\right)$ is weakly contained in the regular representation and that $N$ is amenable. Then the bimodule ${ }_{M} \mathrm{~L}^{2}(\widetilde{M} \ominus M)_{M}$ is weakly contained in the coarse $M-M-$ bimodule.

Proof. Whenever $\eta: \Gamma \rightarrow \mathcal{U}(\mathcal{K})$ is a unitary representation, we define the $M$ - $M$-bimodule $\mathcal{H}^{\eta}$ on the Hilbert space $\mathrm{L}^{2}(M) \otimes \mathcal{K}$ with bimodule structure

$$
\left(a u_{g}\right) \cdot(x \otimes \xi) \cdot\left(b u_{h}\right)=a u_{g} x b u_{h} \otimes \eta(g) \xi \quad \text { for all } \quad a, b \in N, g, h \in \Gamma, x \in M, \xi \in \mathcal{K} .
$$

If $\eta$ is the regular representation, one checks that $\mathcal{H}^{\eta}$ is unitarily equivalent with $\mathrm{L}^{2}(M) \otimes_{N} \mathrm{~L}^{2}(M)$. Because $N$ is amenable we conclude that $\mathcal{H}^{\eta}$ is weakly contained in the coarse bimodule whenever $\eta$ is weakly contained in the regular representation.

One checks that ${ }_{M} \mathrm{~L}^{2}(\widetilde{M} \ominus M)_{M}$ is unitarily equivalent to the direct sum of $\mathcal{H}^{\eta}$ where $\eta$ runs through all the symmetric tensor powers of the complexified $\pi$. Since all these symmetric tensor powers are weakly contained in the regular representation, the lemma follows.

Following Ozawa Oz03, a $\mathrm{II}_{1}$ factor $M$ is called solid if $A^{\prime} \cap M$ is amenable for every diffuse von Neumann subalgebra $A \subset M$. Ozawa proved in Oz03 that all group von Neumann algebras of icc hyperbolic groups are solid. In particular the free group factors $\mathrm{LF}_{n}, n \geq 2$, are solid. Although this is not needed in the rest of this paper, we reprove Peterson's [Pe06, Theorem 1.3] on the solidity of the group factors $L \Gamma$ when $\Gamma$ is an icc group that admits a proper ${ }^{(4)} 1$-cocycle into a multiple of the regular representation. Prior to this Popa had given in Po06b] a new proof for the solidity of the free group factors $\mathrm{LF}_{n}$ using the malleable deformation coming from the word length. Our proof is very close to Popa's, replacing the word length by a proper 1-cocycle.

It is shown in [CSV07] that for every finite group $H$ and $n \geq 2$, the wreath product $\Gamma=H \geq \mathbb{F}_{n}:=$ $H^{\left(\mathbb{F}_{n}\right)} \rtimes \mathbb{F}_{n}$ admits a so-called proper wall structure with the stabilizer of every wall being amenable. Hence $\Gamma$ admits a proper 1-cocycle into an orthogonal representation that is weakly contained in the regular representation. In particular $\mathrm{L} \Gamma$ is solid by Theorem 3.6 below. A more general result is proven by Ozawa in [Oz04, Corollary 4.5] implying that all wreath products $H$ ¿ $\Gamma$ with $H$ amenable and $\Gamma$ hyperbolic, have a solid group von Neumann algebra.

Theorem 3.6 ([e06, Theorem 1.3]). Let $\Gamma$ be an icc group that admits a proper 1-cocycle into an orthogonal representation $\pi$ that is weakly contained in the regular representation. Then $\mathrm{L} \Gamma$ is solid.

Proof. Let $A \subset M$ be a diffuse von Neumann subalgebra and assume by contradiction that $A^{\prime} \cap M$ is nonamenable. So we can take a nonzero projection $p \in \mathcal{Z}\left(A^{\prime} \cap M\right)$ such that $P:=\left(A^{\prime} \cap M\right) p$ has no amenable direct summand.

Let $b: \Gamma \rightarrow H_{\mathbb{R}}$ be a proper 1-cocycle into the orthogonal representation $\pi: \Gamma \rightarrow \mathcal{O}\left(H_{\mathbb{R}}\right)$ that is weakly contained in the regular representation. Consider the one-parameter group of automorphisms $\left(\alpha_{t}\right)$ of $\widetilde{M}=D \rtimes \Gamma$ as above. By Lemma 3.5 the bimodule ${ }_{M} \mathrm{~L}^{2}(\widetilde{M} \ominus M)_{M}$ is weakly contained in the coarse $M$ - $M$-bimodule. By assumption $P$ has no amenable direct summand and we just observed that ${ }_{P} \mathrm{~L}^{2}(p \widetilde{M} p \ominus p M p)_{P}$ is weakly contained in the coarse $P$-P-bimodule. As we

\footnotetext{
${ }^{(4)}$ Recall that a 1 -cocycle $b: \Gamma \rightarrow H_{\mathbb{R}}$ is called proper if $\|b(g)\| \rightarrow \infty$ whenever $g \rightarrow \infty$.
} 
explain for the sake of completeness in Remark 3.7 below, it follows that for every $\varepsilon>0$, there exists a finite subset $\mathcal{F} \subset(P)_{1}$ and a $\delta>0$ such that

$$
\text { if } x \in p(\widetilde{M} \ominus M) p \text { satisfies }\|x\| \leq 2 \text { and }\|[x, y]\|_{2} \leq \delta \text { for all } y \in \mathcal{F} \text { then }\|x\|_{2} \leq \varepsilon .
$$

We prove that $\alpha_{t} \rightarrow$ id uniformly on the unit ball of $A p$. Choose $\varepsilon>0$. Take a finite subset $\mathcal{F} \subset(P)_{1}$ and a $\delta>0$ such that (3.1) holds. Put $\delta_{0}=\min \{\delta / 12, \varepsilon\}$. Take $t>0$ small enough such that $\left\|\alpha_{t}(p)-p\right\|_{2} \leq \delta_{0}$ and $\left\|\alpha_{t}(y)-y\right\|_{2} \leq \delta_{0}$ for all $y \in \mathcal{F}$. We claim that $\left\|\alpha_{t}(a p)-a p\right\|_{2} \leq 5 \sqrt{2} \varepsilon$ for all $a \in(A)_{1}$. Once this claim is proven, we have shown that $\alpha_{t} \rightarrow$ id uniformly on the unit ball of $A p$.

To prove the claim, fix $a \in(A)_{1}$. As before write $\delta_{t}(d)=\alpha_{t}(d)-E_{M}\left(\alpha_{t}(d)\right)$ for all $d \in M$. Define $x=p \delta_{t}(a) p$. Since $\left\|\alpha_{t}(p)-p\right\|_{2} \leq \delta_{0}$, it follows that $\left\|p \alpha_{t}(a) p-\alpha_{t}(a p)\right\|_{2} \leq 2 \delta_{0}$ and hence $\left\|x-\delta_{t}(a p)\right\|_{2} \leq 4 \delta_{0}$. Since $\alpha_{t}(a p)$ and $\alpha_{t}(y)$ commute for all $y \in \mathcal{F}$, it follows that $\left\|\left[\alpha_{t}(a p), y\right]\right\|_{2} \leq$ $2 \delta_{0}$ and hence $\left\|\left[\delta_{t}(a p), y\right]\right\|_{2} \leq 4 \delta_{0}$ for all $y \in \mathcal{F}$. So, $\|[x, y]\|_{2} \leq 12 \delta_{0} \leq \delta$ for all $y \in \mathcal{F}$. Since $\|x\| \leq 2$ we conclude from (3.1) that $\|x\|_{2} \leq \varepsilon$. Hence, $\left\|\delta_{t}(a p)\right\|_{2} \leq \varepsilon+4 \delta_{0} \leq 5 \varepsilon$. By Lemma 3.1 we get that $\left\|\alpha_{t}(a p)-a p\right\|_{2} \leq 5 \sqrt{2} \varepsilon$.

So $\alpha_{t} \rightarrow$ id uniformly on the unit ball of $A p$. Using the facts that $A p$ is diffuse and that the 1-cocycle $b$ is proper, we finally derive a contradiction. Since $\alpha_{t} \rightarrow$ id uniformly on $\mathcal{U}(A p)$, we can fix $t>0$ such that $\left\|E_{M}\left(\alpha_{t}(w p)\right)\right\|_{2} \geq\|p\|_{2} / 2$ for all $w \in \mathcal{U}(A)$. Since $A$ is diffuse, we can take a sequence of unitaries $w_{n} \in \mathcal{U}(A)$ tending to zero weakly. We prove that $\left\|E_{M}\left(\alpha_{t}\left(w_{n} p\right)\right)\right\|_{2} \rightarrow 0$ as $n \rightarrow \infty$. Let $w_{n} p=\sum_{g \in \Gamma} \lambda_{g}^{n} u_{g}$ be the Fourier expansion of $w_{n} p$, with $\lambda_{g}^{n} \in \mathbb{C}$. Since $w_{n} p \rightarrow 0$ weakly, for every fixed $g \in \Gamma$ we have $\lambda_{g}^{n} \rightarrow 0$ as $n \rightarrow \infty$. Fix $\varepsilon>0$. Take a finite subset $\mathcal{F} \subset \Gamma$ such that $\exp \left(-2 t^{2}\|b(g)\|^{2}\right)<\varepsilon$ for all $g \in \Gamma-\mathcal{F}$. Take $n_{0} \in \mathbb{N}$ such that $\sum_{g \in \mathcal{F}}\left|\lambda_{g}^{n}\right|^{2}<\varepsilon$ for all $n \geq n_{0}$. So for all $n \geq n_{0}$ we have

$$
\left\|E_{M}\left(\alpha_{t}\left(w_{n} p\right)\right)\right\|_{2}^{2}=\sum_{g \in \Gamma} \exp \left(-2 t^{2}\|b(g)\|^{2}\right)\left|\lambda_{g}^{n}\right|^{2} \leq \sum_{g \in \mathcal{F}}\left|\lambda_{g}^{n}\right|^{2}+\sum_{g \in \Gamma-\mathcal{F}} \varepsilon\left|\lambda_{g}^{n}\right|^{2}<\varepsilon+\varepsilon\left\|w_{n} p\right\|_{2}^{2} \leq 2 \varepsilon .
$$

Hence we have shown that $\left\|E_{M}\left(\alpha_{t}\left(w_{n} p\right)\right)\right\|_{2} \rightarrow 0$ as $n \rightarrow \infty$ which is a contradiction with the fact that $\left\|E_{M}\left(\alpha_{t}(w p)\right)\right\|_{2} \geq\|p\|_{2} / 2$ for all $w \in \mathcal{U}(A)$.

Remark 3.7. The following is a detailed explanation for (3.1). Let $(P, \tau)$ be a tracial von Neumann algebra and ${ }_{P} \mathcal{H}_{P}$ a $P$-P-bimodule. Assume that $\xi_{n} \in \mathcal{H}$ is a sequence of vectors and $\kappa, \varepsilon>0$ are positive numbers such that

$$
\begin{aligned}
& \left\|\xi_{n}\right\| \geq \varepsilon \text { for all } n \in \mathbb{N}, \quad\left\langle x \xi_{n}, \xi_{n}\right\rangle \leq \kappa \tau(x) \text { for all } x \in P^{+} \text {and } \\
& \lim _{n}\left\|x \xi_{n}-\xi_{n} x\right\|=0 \text { for all } x \in P .
\end{aligned}
$$

We claim that there is a nonzero central projection $z \in \mathcal{Z}(P)$ such that $z \mathcal{H} z$ weakly contains the trivial bimodule ${ }_{P z} \mathrm{~L}^{2}(P z)_{P z}$. By our assumptions we find $T_{n} \in P^{+}$such that $\left\|T_{n}\right\| \leq \kappa$ and $\left\langle x \xi_{n}, \xi_{n}\right\rangle=\tau\left(x T_{n}\right)$ for all $x \in P$. After a passage to a subsequence we may assume that $T_{n} \rightarrow T$ weakly. Since $\left\|\xi_{n}\right\| \geq \varepsilon$ for all $n \in \mathbb{N}$ we have $\tau(T) \geq \varepsilon^{2}$. So $T \neq 0$. Because $\lim _{n}\left\|x \xi_{n}-\xi_{n} x\right\|=0$ for all $x \in P$, it follows that $T \in \mathcal{Z}(P)$. Since $T$ is positive, take a nonzero central projection $z \in \mathcal{Z}(P)$ and an element $S \in \mathcal{Z}(P)^{+}$such that $S^{2} T=z$. Write $\eta_{n}=S \xi_{n}$. It follows that

$$
\left\langle x \eta_{n} y, a \eta_{n} b\right\rangle \rightarrow \tau\left(x y b^{*} a^{*}\right)=\langle x y, a b\rangle \text { for all } x, y, a, b \in P z .
$$

This proves our claim. 


\subsection{Cocycles into representations that are mixing relative to a family of sub- groups}

Assume that $\pi$ is mixing relative to a family $\mathcal{S}$ of subgroups of $\Gamma$. As before we say that a subset $\mathcal{F} \subset \Gamma$ is small relative to $\mathcal{S}$ if $\mathcal{F}$ can be written as a finite union of $g \Sigma h, g, h \in \Gamma, \Sigma \in \mathcal{S}$. Denote by $P_{\mathcal{F}}$ the orthogonal projection of $\mathrm{L}^{2}(M)$ onto the closed linear span of $\left\{a u_{g} \mid a \in N, g \in \mathcal{F}\right\}$.

We prove the following technical lemma. It will be useful in Section 4, but also when proving Proposition 3.9 and Theorem 3.10 below. The final statement of Lemma 3.8 'controls' the normalizer of certain von Neumann subalgebras $B \subset p \widetilde{M} p$. The usage of mixing techniques to control normalizers goes back to Popa's [Po03, Section 3].

Lemma 3.8. Assume that $\pi$ is mixing relative to a family $\mathcal{S}$ of subgroups of $\Gamma$. Use the notation $P_{\mathcal{F}}$ as explained before the lemma.

Let $K \subseteq D \ominus \mathbb{C} 1$ be a finite dimensional vector subspace. Denote by $Q_{K}$ the orthogonal projection of $\mathrm{L}^{2}(\widetilde{M})$ onto the closed linear span of $(d \otimes a) u_{g}, d \in K, a \in N, g \in \Gamma$. Note that $Q_{K}$ is right $M$-modular.

For every finite dimensional subspace $K \subset D \ominus \mathbb{C} 1$, every $x \in(\widetilde{M})_{1}$ and every $\varepsilon>0$, there exists a subset $\mathcal{F} \subset \Gamma$ that is small relative to $\mathcal{S}$ such that

$$
\left\|Q_{K}(v x)\right\|_{2} \leq\left\|P_{\mathcal{F}}(v)\right\|_{2}+\varepsilon \quad \text { for all } \quad v \in(M)_{1} .
$$

In particular, if $p \in M$ is a projection and $B \subset p M p$ is a von Neumann subalgebra satisfying $B \nprec N \rtimes \Sigma$ for all $\Sigma \in \mathcal{S}$, then the normalizer of $B$ inside $p \widetilde{M} p$ is contained in $p M p$.

Proof. Fix the finite dimensional subspace $K \subset D \ominus \mathbb{C} 1$, the element $x \in(\widetilde{M})_{1}$ and $\varepsilon>0$. By the Kaplansky density theorem we can take $d_{1}, \ldots, d_{n} \in D$ and $z_{1}, \ldots, z_{n} \in M$ such that the element

$$
x_{0}:=\sum_{i=1}^{n}\left(d_{i} \otimes 1\right) z_{i}
$$

satisfies $\left\|x_{0}\right\| \leq 1$ and $\left\|x-x_{0}\right\|_{2} \leq \varepsilon / 2$. Put $\kappa=\max \left\{\left\|z_{1}\right\|, \ldots,\left\|z_{n}\right\|\right\}$. Note that $\left(\sigma_{g}\right)_{g \in \Gamma}$ viewed as a unitary representation of $\Gamma$ on $\mathrm{L}^{2}(D \ominus \mathbb{C} 1)$, is mixing relative to $\mathcal{S}$. So we can take a subset $\mathcal{F} \subset \Gamma$ that is small relative to $\mathcal{S}$ such that

$$
\left\|Q_{K}\left(\sigma_{g}\left(d_{i}\right)\right)\right\|_{2} \leq \frac{\varepsilon}{2 n \kappa} \quad \text { for all } \quad g \in \Gamma-\mathcal{F} .
$$

We claim that $\mathcal{F}$ satisfies the conclusion of the lemma. So, take $v \in(M)_{1}$. We have to prove that $\left\|Q_{K}(v x)\right\|_{2} \leq\left\|P_{\mathcal{F}}(v)\right\|_{2}+\varepsilon$.

Since $\left\|v x-v x_{0}\right\|_{2} \leq \varepsilon / 2$, also $\left\|Q_{K}(v x)-Q_{K}\left(v x_{0}\right)\right\|_{2} \leq \varepsilon / 2$ and it suffices to prove that $\left\|Q_{K}\left(v x_{0}\right)\right\|_{2} \leq$ $\left\|P_{\mathcal{F}}(v)\right\|_{2}+\varepsilon / 2$. Let $v=\sum_{g \in \Gamma} v_{g} u_{g}$, with $v_{g} \in N$, be the Fourier expansion of $v$. A direct computation yields that

$$
Q_{K}\left(v x_{0}\right)=\sum_{i=1}^{n} \sum_{g \in \Gamma}\left(Q_{K}\left(\sigma_{g}\left(d_{i}\right)\right) \otimes v_{g}\right) u_{g} z_{i} .
$$


For every $i=1, \ldots, n$, we have

$$
\begin{aligned}
\left\|\sum_{g \in \Gamma-\mathcal{F}}\left(Q_{K}\left(\sigma_{g}\left(d_{i}\right)\right) \otimes v_{g}\right) u_{g} z_{i}\right\|_{2}^{2} & \leq\left\|\sum_{g \in \Gamma-\mathcal{F}}\left(Q_{K}\left(\sigma_{g}\left(d_{i}\right)\right) \otimes v_{g}\right) u_{g}\right\|_{2}^{2}\left\|z_{i}\right\|^{2} \\
& =\left\|z_{i}\right\|^{2} \sum_{g \in \Gamma-\mathcal{F}}\left\|Q_{K}\left(\sigma_{g}\left(d_{i}\right)\right)\right\|_{2}^{2}\left\|v_{g}\right\|_{2}^{2} \\
& \leq\left(\frac{\varepsilon\left\|z_{i}\right\|}{2 n \kappa}\right)^{2} \sum_{g \in \Gamma-\mathcal{F}}\left\|v_{g}\right\|_{2}^{2} \leq\left(\frac{\varepsilon}{2 n}\right)^{2} .
\end{aligned}
$$

Hence,

$$
\left\|\sum_{i=1}^{n} \sum_{g \in \Gamma-\mathcal{F}}\left(Q_{K}\left(\sigma_{g}\left(d_{i}\right)\right) \otimes v_{g}\right) u_{g} z_{i}\right\|_{2} \leq \frac{\varepsilon}{2} .
$$

On the other hand,

$$
\sum_{i=1}^{n} \sum_{g \in \mathcal{F}}\left(Q_{K}\left(\sigma_{g}\left(d_{i}\right)\right) \otimes v_{g}\right) u_{g} z_{i}=Q_{K}\left(P_{\mathcal{F}}(v) x_{0}\right)
$$

and $\left\|Q_{K}\left(P_{\mathcal{F}}(v) x_{0}\right)\right\|_{2} \leq\left\|P_{\mathcal{F}}(v) x_{0}\right\|_{2} \leq\left\|P_{\mathcal{F}}(v)\right\|_{2}$. Altogether we have shown that $\left\|Q_{K}\left(v x_{0}\right)\right\|_{2} \leq$ $\left\|P_{\mathcal{F}}(v)\right\|_{2}+\varepsilon / 2$.

Finally assume that $B \subset p M p$ is a von Neumann subalgebra satisfying $B \nprec N \rtimes \Sigma$ for all $\Sigma \in \mathcal{S}$. Lemma 2.4 provides a net $\left(v_{i}\right)$ of unitaries in $B$ such that $\left\|P_{\mathcal{F}}\left(v_{i}\right)\right\|_{2} \rightarrow 0$ for every subset $\mathcal{F} \subset \Gamma$ that is small relative to $\mathcal{S}$. Let $x \in \mathcal{N}_{p \widetilde{M} p}(B)$. We have to prove that $x \in M$. Let $K \subset D \ominus \mathbb{C} 1$ be an arbitrary finite dimensional subspace. It suffices to prove that $Q_{K}(x)=0$. Let $\varepsilon>0$. Take a subset $\mathcal{F} \subset \Gamma$ that is small relative to $\mathcal{S}$ and such that

$$
\left\|Q_{K}(v x)\right\|_{2} \leq\left\|P_{\mathcal{F}}(v)\right\|_{2}+\varepsilon
$$

for all $v \in(M)_{1}$. Taking $v=v_{i}$ and using that $Q_{K}$ is right $M$-modular, it follows that

$$
\left\|Q_{K}(x)\right\|_{2}=\left\|Q_{K}(x) x^{*} v_{i} x\right\|_{2}=\left\|Q_{K}\left(v_{i} x\right)\right\|_{2} \leq\left\|P_{\mathcal{F}}\left(v_{i}\right)\right\|_{2}+\varepsilon \rightarrow \varepsilon .
$$

Hence $\left\|Q_{K}(x)\right\|_{2} \leq \varepsilon$ for every $\varepsilon>0$. So $Q_{K}(x)=0$.

Proposition 3.9. Assume that $\pi$ is mixing relative to a family $\mathcal{S}$ of subgroups of $\Gamma$ and that $b$ is bounded on every $\Sigma \in \mathcal{S}$. Let $p \in M$ be a projection and $B \subset p M p$ a von Neumann subalgebra generated by a group of unitaries $\mathcal{G} \subset \mathcal{U}(B)$. Denote by $Q$ the normalizer of $B$ inside $p M p$.

Let $q \in \mathcal{Z}(Q)$ be the maximal projection given by Lemma 3.3 such that $\alpha_{t} \rightarrow$ id in $\|\cdot\|_{2}$-norm uniformly on the unit ball of Bq. There exists a sequence of unitaries $\left(w_{n}\right)$ in $\mathcal{G}$ such that for every $t>0$ we have that $\left\|E_{M}\left(\alpha_{t}\left(w_{n}(p-q)\right)\right)\right\|_{2} \rightarrow 0$ as $n \rightarrow \infty$.

Proof. By Proposition 2.6 let $q_{0} \in \mathcal{Z}(Q)$ be the maximal projection such that $\left(B q_{0}\right)_{1} \subset$ approx $N \rtimes \mathcal{S}$. Since $b$ is bounded on every subgroup $\Sigma \in \mathcal{S}$ and hence on every subset $\mathcal{F} \subset \Gamma$ that is small relative to $\mathcal{S}$, one checks easily that $\alpha_{t} \rightarrow$ id in $\|\cdot\|_{2}$-norm uniformly on the unit ball of $B q_{0}$. So in order to prove the proposition we may replace $p$ by $p-q_{0}$ and $B$ by $B\left(p-q_{0}\right)$ and assume that $B \nprec N \rtimes \Sigma$ for all $\Sigma \in \mathcal{S}$.

Assume that there is no sequence of unitaries $\left(w_{n}\right)$ in $\mathcal{G}$ such that $\left\|E_{M}\left(\alpha_{t}\left(w_{n}(p-q)\right)\right)\right\|_{2} \rightarrow 0$ for every $t>0$. So, we find $s, \delta>0$ such that $\left\|E_{M}\left(\alpha_{s}(b(p-q))\right)\right\|_{2} \geq \delta$ for all $b \in \mathcal{G}$. Put $t=\sqrt{2} s$. This means that

$$
\tau\left(b^{*}(p-q) \alpha_{t}((p-q) b)\right)=\left\|E_{M}\left(\alpha_{s}(b(p-q))\right)\right\|_{2}^{2} \geq \delta^{2} \quad \text { for all } b \in \mathcal{G} .
$$


Hence the element $w \in \widetilde{M}$ of minimal $\|\cdot\|_{2}$-norm in the weakly closed convex hull of

$$
\left\{b^{*}(p-q) \alpha_{t}((p-q) b) \mid b \in \mathcal{G}\right\}
$$

is nonzero and satisfies $b w=w \alpha_{t}(b)$ for all $b \in B$ and $w^{*}=\alpha_{t}(\beta(w))$. Here $\beta$ denotes the automorphism of $\widetilde{M}$ satisfying $\beta(x)=x$ for all $x \in M$ and $\beta(\omega(\xi))=\omega(\xi)^{*}$ for all $\xi \in H_{\mathbb{R}}$. Recall that $\beta \circ \alpha_{t}=\alpha_{-t} \circ \beta$. Also, by construction $q w=0$. We shall prove that the support projection $r$ of $w w^{*}$ satisfies $r \leq q$, hence reaching a contradiction. So we have to prove that $r \in B^{\prime} \cap p M p$ and that $\alpha_{t} \rightarrow$ id uniformly on $(B)_{1} r$.

Let $v$ be the polar part of $w$. It follows that $v v^{*}=r$ and $b v=v \alpha_{t}(b)$ for all $b \in B$. It also follows that $v^{*} v$ is the support projection of $w^{*} w$. Since $w^{*}=\alpha_{t}(\beta(w))$, we have $w^{*} w=\alpha_{t}\left(\beta\left(w w^{*}\right)\right)$ and conclude that $v^{*} v=\alpha_{t}(\beta(r))$. By Lemma 3.8 and because $B \nprec N \rtimes \Sigma$ for all $\Sigma \in \mathcal{S}$, we get that $B^{\prime} \cap p \widetilde{M} p=B^{\prime} \cap p M p$. So, $r \in B^{\prime} \cap p M p$. Since $\beta(x)=x$ for all $x \in M$, we have in particular that $\beta(r)=r$. We have seen above that $v^{*} v=\alpha_{t}(\beta(r))$ and hence $v^{*} v=\alpha_{t}(r)$.

Choose $1 \geq \varepsilon>0$. By Lemma 3.2 take $t^{\prime}>0$ such that

$$
\left\|E_{M}\left(v \alpha_{t}(a) v^{*}\right)\right\|_{2} \leq\left\|E_{M}\left(\alpha_{t^{\prime}}(a)\right)\right\|_{2}+\varepsilon \text { for all } a \in(M)_{1} .
$$

Applying this to $a=b r$ for $b \in(B)_{1}$ it follows that $\|b r\|_{2}-\| E_{M}\left(\alpha_{t^{\prime}}(b r) \|_{2} \leq \varepsilon\right.$ for all $b \in(B)_{1}$. So we have shown that $\|b r\|_{2}-\left\|E_{M}\left(\alpha_{t}(b r)\right)\right\|_{2} \rightarrow 0$ uniformly in $b \in(B)_{1}$. This precisely means that $\alpha_{t} \rightarrow$ id in $\|\cdot\|_{2}$-norm uniformly on $(B)_{1} r$.

\subsection{From uniform convergence on $B$ to uniform convergence on the normalizer}

In [Pe06, Theorem 4.5] and [CP10, Theorem 2.5] Peterson and Chifan-Peterson prove that if $B \subset$ $p M p$ is a von Neumann subalgebra such that $\alpha_{t} \rightarrow$ id uniformly on the unit ball of $B$ and such that for all $\Sigma \in \mathcal{S}$ we have $B \nprec N \rtimes \Sigma$, then $\alpha_{t} \rightarrow$ id uniformly on the unit ball of the normalizer of $B$. They prove this theorem using the technology of unbounded derivations. We repeat their proof, but using the malleable dilation technology from paragraph 3.1 , which makes things slightly easier.

Theorem 3.10 ([י $\mathrm{Pe} 06$, Theorem 4.5] and [CP10, Theorem 2.5]). Assume that $\pi$ is mixing relative to a family $\mathcal{S}$ of subgroups of $\Gamma$. Let $p \in M$ be a projection and $B \subset p M p$ a von Neumann subalgebra. Let $r \in B^{\prime} \cap p M p$ be a projection and make the following assumptions.

- If $t \rightarrow 0$ then $\alpha_{t} \rightarrow$ id in $\|\cdot\|_{2}$-norm uniformly on $(B r)_{1}$.

- For every $\Sigma \in \mathcal{S}$ we have $B r \nprec N \rtimes \Sigma$.

Denote by $Q$ the normalizer of $B$ inside $p M p$. Define $q$ as the smallest projection in $\mathcal{Z}(Q)$ satisfying $r \leq q$. Then $\alpha_{t} \rightarrow$ id in $\|\cdot\|_{2}$-norm uniformly on $(Q q)_{1}$.

Proof. From Lemma 2.4 and Proposition 2.6 it follows that $B q \nprec N \rtimes \Sigma$ for all $\Sigma \in \mathcal{S}$ and that we can take a net of unitaries $\left(v_{i}\right)_{i \in I}$ in $\mathcal{U}(B)$ such that $\lim _{i}\left\|P_{\mathcal{F}}\left(v_{i} q\right)\right\|_{2}=0$ for every subset $\mathcal{F} \subset \Gamma$ that is small relative to $\mathcal{S}$. By Lemma 3.3 we have $\alpha_{t} \rightarrow$ id in $\|\cdot\|_{2}$-norm uniformly on the unit ball of $B q$.

For every $x \in M$, put $\delta_{t}(x)=\alpha_{t}(x)-E_{M}\left(\alpha_{t}(x)\right)$. By Lemma 3.1 and Proposition 3.4 it suffices to prove that $\delta_{t} \rightarrow 0$ in $\|\cdot\|_{2}$-norm uniformly on $\mathcal{N}_{Q}(B) q$. Choose $\varepsilon>0$. Put $\delta=\varepsilon^{2} /(4 \tau(q))$. 
Take $t>0$ such that $\left\|\alpha_{t}(b q)-b q\right\|_{2} \leq \delta\|q\|_{2}$ for all $b \in \mathcal{U}(B)$. We show that $\left\|\delta_{t}(u q)\right\|_{2} \leq \varepsilon$ for all $u \in \mathcal{N}_{Q}(B)$, hence proving the claim. To prove this statement, fix $u \in \mathcal{N}_{Q}(B)$. Using the notation in Lemma 3.8 take a finite dimensional subspace $K \subset D \ominus \mathbb{C} 1$ such that $\left\|\left(1-Q_{K}\right) \delta_{t}(u q)\right\|_{2} \leq \delta\|q\|_{2}$. By Lemma 3.8 take a subset $\mathcal{F} \subset \Gamma$ that is small relative to $\mathcal{S}$ and such that $\left\|Q_{K}\left(v \alpha_{t}(u)\right)\right\|_{2} \leq$ $\left\|P_{\mathcal{F}}(v)\right\|_{2}+\delta\|q\|_{2}$ for all $v$ in the unit ball of $M$.

Write $d_{i}:=u^{*} v_{i}^{*} u$ and note that $d_{i} \in \mathcal{U}(B)$. By construction $u q=v_{i} q u d_{i} q$ for all $i \in I$. By our choice of $t$ we have $\left\|\alpha_{t}\left(v_{i} q\right)-v_{i} q\right\|_{2} \leq \delta\|q\|_{2}$ and $\left\|\alpha_{t}\left(d_{i} q\right)-d_{i} q\right\|_{2} \leq \delta\|q\|_{2}$ for all $i \in I$. Using the fact that $Q_{K}$ is right $M$-modular, it follows that for all $i \in I$,

$$
\begin{aligned}
\left\|\delta_{t}(u q)\right\|_{2}^{2} & =\left\langle\alpha_{t}(u q), \delta_{t}(u q)\right\rangle=\left\langle\alpha_{t}\left(v_{i} q u d_{i} q\right), \delta_{t}(u q)\right\rangle \\
& \leq\left|\left\langle v_{i} q \alpha_{t}(u) d_{i} q, \delta_{t}(u q)\right\rangle\right|+2 \delta \tau(q) \leq\left|\left\langle v_{i} q \alpha_{t}(u) d_{i} q, Q_{K}\left(\delta_{t}(u q)\right)\right\rangle\right|+3 \delta \tau(q) \\
& =\left|\left\langle Q_{K}\left(v_{i} q \alpha_{t}(u) d_{i} q\right), \delta_{t}(u q)\right\rangle\right|+3 \delta \tau(q) \leq\left\|Q_{K}\left(v_{i} q \alpha_{t}(u)\right) d_{i} q\right\|_{2}\|q\|_{2}+3 \delta \tau(q) \\
& \leq\left\|Q_{K}\left(v_{i} q \alpha_{t}(u)\right)\right\|_{2}\|q\|_{2}+3 \delta \tau(q) \leq\left\|P_{\mathcal{F}}\left(v_{i} q\right)\right\|_{2}\|q\|_{2}+4 \delta \tau(q) .
\end{aligned}
$$

Taking the limit over $i \in I$ it follows that $\left\|\delta_{t}(u q)\right\|_{2}^{2} \leq 4 \delta \tau(q)=\varepsilon^{2}$. We have shown that $\delta_{t} \rightarrow 0$ in $\|\cdot\|_{2}$-norm uniformly on $\mathcal{N}_{Q}(B) q$.

\section{Deformations must be uniform when they are uniform on enough normalizing unitaries}

Let $\Gamma \curvearrowright(N, \tau)$ be a trace preserving action and put $M=N \rtimes \Gamma$. Assume that $\pi: \Gamma \rightarrow \mathcal{O}\left(H_{\mathbb{R}}\right)$ is an orthogonal representation that is mixing relative to a family $\mathcal{S}$ of subgroups of $\Gamma$. Let $b: \Gamma \rightarrow H_{\mathbb{R}}$ be a 1-cocycle w.r.t. $\pi$ and assume that $b$ is bounded on every $\Sigma \in \mathcal{S}$. As above we call a subset $\mathcal{F} \subset \Gamma$ small relative to $\mathcal{S}$ if $\mathcal{F}$ can be written as a finite union of subsets of the form $g \Sigma h, g, h \in \Gamma$, $\Sigma \in \mathcal{S}$. Whenever $\mathcal{F} \subset \Gamma$ we denote by $P_{\mathcal{F}}$ the orthogonal projection of $\mathrm{L}^{2}(M)$ onto the closed linear span of $\left\{a u_{g} \mid a \in N, g \in \Gamma\right\}$.

Consider the algebra $\widetilde{M} \supset M$ together with the 1-parameter group of automorphisms $\left(\alpha_{t}\right)$ of $\widetilde{M}$ as in paragraph 3.1. We say that $\alpha_{t} \rightarrow$ id in $\|\cdot\|_{2}$-norm uniformly on the tail of a net $\left(v_{i}\right)_{i \in I}$ if for every $\varepsilon>0$, there exists a $t_{0}>0$ and $i_{0} \in I$ such that $\left\|v_{i}-\alpha_{t}\left(v_{i}\right)\right\|_{2}<\varepsilon$ for all $i \geq i_{0}$ and all $|t|<t_{0}$.

In this section we prove the following result: if $B \subset p M p$ is an abelian von Neumann subalgebra that is normalized by 'enough' unitaries $\left(v_{i}\right)$ such that $\alpha_{t} \rightarrow$ id uniformly on the tail of $\left(v_{i}\right)$, then $\alpha_{t} \rightarrow$ id uniformly on the unit ball of $B$. In the special where $\mathcal{S}$ consists of normal subgroups of $\Gamma$ and using the technology of unbounded derivations and ultrapowers, this theorem was first proven by Chifan-Peterson in [CP10, Theorem 3.2] and several of the ideas go back to Peterson's proof of [Pe09, Theorem 4.1].

Theorem 4.1. Let $p \in M$ be a projection and $B \subset p M p$ an abelian von Neumann subalgebra that is normalized by a net of unitaries $\left(v_{i}\right)_{i \in I}$ in $\mathcal{U}(p M p)$. Let $r \in p M p$ be any projection and make the following assumptions.

- If $t \rightarrow 0$ then $\alpha_{t} \rightarrow$ id in $\|\cdot\|_{2}$-norm uniformly on the tail of $\left(v_{i}\right)_{i \in I}$.

- For every subset $\mathcal{F} \subset \Gamma$ that is small relative to $\mathcal{S}$, we have $\lim _{i}\left\|P_{\mathcal{F}}\left(v_{i} r\right)\right\|_{2}=0$.

Denote by $Q$ the normalizer of $B$ inside $p M p$ and define $q$ as the smallest projection in $\mathcal{Z}(Q)$ that satisfies $r \leq q$. Then $\alpha_{t} \rightarrow$ id in $\|\cdot\|_{2}$-norm uniformly on $(B q)_{1}$. 
To prove this theorem we need two technical lemmas.

Lemma 4.2. Assume that $\left(v_{i}\right)_{i \in I}$ and $\left(w_{i}\right)_{i \in I}$ are bounded nets in $M$ such that $\left\|P_{\mathcal{F}}\left(v_{i}\right)\right\|_{2} \rightarrow 0$ and $\left\|P_{\mathcal{F}}\left(w_{i}\right)\right\|_{2} \rightarrow 0$ for all subsets $\mathcal{F} \subset \Gamma$ that are small relative to $\mathcal{S}$. Then,

$$
\left\|E_{M}\left(x v_{i} y w_{i} z\right)\right\|_{2} \rightarrow 0 \quad \text { for all } y \in \widetilde{M} \ominus M, x, z \in \widetilde{M} .
$$

Proof. We can approximate $x, y, z$ by linear combinations of $(\omega(\xi) \otimes 1) a, \xi \in H_{\mathbb{R}}, a \in M$. If the nets $v_{i}, w_{i}$ satisfy the hypotheses of the lemma, the same is true, using Lemma 2.3 , for the nets $a v_{i} b$ and $a w_{i} b$, given fixed elements $a, b \in M$. As a result it suffices to prove the lemma when

$$
x=\omega\left(\xi_{1}\right) \otimes 1, \quad y=\left(\omega\left(\xi_{2}\right)-\exp \left(-\left\|\xi_{2}\right\|^{2}\right) 1\right) \otimes 1, \quad z=\omega\left(\xi_{3}\right) \otimes 1
$$

and $\xi_{1}, \xi_{2}, \xi_{3} \in H_{\mathbb{R}}$. In that case a direct computation yields

$$
E_{M}\left(x v_{i} y w_{i} z\right)=\exp \left(-\left\|\xi_{1}\right\|^{2}-\left\|\xi_{2}\right\|^{2}-\left\|\xi_{3}\right\|^{2}\right) \varphi_{31}\left(\varphi_{21}\left(v_{i}\right) \varphi_{32}\left(w_{i}\right)-v_{i} w_{i}\right)
$$

where for all $\alpha, \beta \in\{1,2,3\}$ we define the completely bounded maps $\varphi_{\alpha \beta}: M \rightarrow M$ given by

$$
\varphi_{\alpha \beta}\left(a u_{g}\right)=\exp \left(-2\left\langle\pi(g) \xi_{\alpha}, \xi_{\beta}\right\rangle\right) a u_{g} \quad \text { for all } a \in N, g \in \Gamma \text {. }
$$

One checks that $\varphi_{\alpha \beta}(x)=\exp \left(\left\|\xi_{\alpha}\right\|^{2}+\left\|\xi_{\beta}\right\|^{2}\right) E_{M}\left(\omega\left(\xi_{\alpha}\right) x \omega\left(\xi_{\beta}\right)\right)$ for all $x \in M$, implying that the $\varphi_{\alpha \beta}$ are indeed well defined completely bounded maps.

By our assumptions on $v_{i}$ and $w_{i}$, we have for all $\alpha, \beta \in\{1,2,3\}$ that $\left\|\varphi_{\alpha \beta}\left(v_{i}\right)-v_{i}\right\|_{2} \rightarrow 0$. Since $\left(\varphi_{\alpha \beta}\left(v_{i}\right)\right)_{i}$ and $\left(\varphi_{\alpha \beta}\left(w_{i}\right)\right)_{i}$ are moreover bounded nets, it follows that

$$
\left\|\varphi_{21}\left(v_{i}\right) \varphi_{32}\left(w_{i}\right)-v_{i} w_{i}\right\|_{2} \rightarrow 0 .
$$

But then also $\left\|E_{M}\left(x v_{i} y w_{i} z\right)\right\|_{2} \rightarrow 0$.

In order to prove 4.2 we only used that $\pi$ is mixing relative to the family $\mathcal{S}$ of subgroups of $\Gamma$. We now also use that the 1 -cocycle $b$ is bounded on every $\Sigma \in \mathcal{S}$.

Lemma 4.3. Let $v_{i}$ and $w_{i}$ be bounded nets in $M$ such that $\left\|P_{\mathcal{F}}\left(v_{i}\right)\right\|_{2} \rightarrow 0$ and $\left\|P_{\mathcal{F}}\left(w_{i}\right)\right\|_{2} \rightarrow 0$ for all subsets $\mathcal{F} \subset \Gamma$ that are small relative to $\mathcal{S}$.

Let $x \in \widetilde{M} \ominus M, t>0$ and $\mathcal{F} \subset \Gamma$ a subset that is small relative to $\mathcal{S}$. Then,

$$
\left\langle v_{i} x w_{i}, \alpha_{t}\left(P_{\mathcal{F}}(\xi)\right)\right\rangle \rightarrow 0 \quad \text { uniformly in } \quad \xi \in \mathrm{L}^{2}(M),\|\xi\|_{2} \leq 1 .
$$

Proof. Fix $x \in \widetilde{M} \ominus M, t>0$ and a subset $\mathcal{F} \subset \Gamma$ that is small relative to $\mathcal{S}$. Write $\mathcal{F}=\bigcup_{k=1}^{m} g_{k} \Sigma_{k} h_{k}$ with $\Sigma_{k} \in \mathcal{S}$. We claim that there exist unitary elements $V_{k}, W_{k} \in \widetilde{M}$ such that

$$
\left|\left\langle v x w, \alpha_{t}\left(P_{\mathcal{F}}(\xi)\right)\right\rangle\right| \leq \sum_{k=1}^{m}\left\|E_{M}\left(V_{k} v x w W_{k}\right)\right\|_{2}
$$

for all $v, w \in M$ and all $\xi \in \mathrm{L}^{2}(M)$ with $\|\xi\|_{2} \leq 1$. Once this claim is proven, the lemma follows from Lemma 4.2 .

Since the 1-cocycle $b$ is bounded on $\Sigma_{k}$, we can take $\eta_{k} \in H_{\mathbb{R}}$ such that $t b(g)=\pi(g) \eta_{k}-\eta_{k}$ for all $g \in \Sigma_{k}$. Note that $\alpha_{t}(x)=\omega\left(\eta_{k}\right)^{*} x \omega\left(\eta_{k}\right)$ for all $x \in \mathrm{L}^{2}\left(N \rtimes \Sigma_{k}\right)$. Put $V_{k}=\omega\left(\eta_{k}\right) \alpha_{t}\left(u_{g_{k}}^{*}\right)$ and $W_{k}=\omega\left(\eta_{k}\right)^{*} \alpha_{t}\left(u_{h_{k}}^{*}\right)$. 
Take $\xi \in \mathrm{L}^{2}(M),\|\xi\|_{2} \leq 1$ arbitrarily. Let $\mathcal{F}_{k} \subset \Sigma_{k}$ be such that $\bigcup_{k=1}^{m} g_{k} \Sigma_{k} h_{k}=\bigsqcup_{k=1}^{m} g_{k} \mathcal{F}_{k} h_{k}$. Define $\xi_{k}=P_{\mathcal{F}_{k}}\left(u_{g_{k}}^{*} \xi u_{h_{k}}^{*}\right)$. Note that $\xi_{k} \in \mathrm{L}^{2}\left(N \rtimes \Sigma_{k}\right)$, that $\left\|\xi_{k}\right\|_{2} \leq 1$ and that we have an orthogonal decomposition

$$
P_{\mathcal{F}}(\xi)=\sum_{k=1}^{n} u_{g_{k}} \xi_{k} u_{h_{k}}
$$

For all $k=1, \ldots, m$, we have

$$
\left|\left\langle v x w, \alpha_{t}\left(u_{g_{k}} \xi_{k} u_{h_{k}}\right)\right\rangle=\right|\left\langle E_{M}\left(V_{k} v x w W_{k}\right), \xi_{k}\right\rangle \mid \leq\left\|E_{M}\left(V_{k} v x w W_{k}\right)\right\|_{2} .
$$

Summing over $k$ yields the claim and hence proves the lemma.

\section{Proof of Theorem 4.1}

Denote by $Q$ the normalizer of $B$ inside $p M p$. Denote by $q_{1} \in \mathcal{Z}(Q)$ the maximal projection given by Lemma 3.3 such that $\alpha_{t} \rightarrow$ id in $\|\cdot\|_{2}$-norm uniformly on the unit ball of $B q_{1}$. If $r \leq q_{1}$, then also $q \leq q_{1}$ and we are done. So assume that $r \not \leq q_{1}$.

Put $T:=\left(p-q_{1}\right) r\left(p-q_{1}\right)$ and note that $T$ is nonzero. Let $r_{0}$ be a nonzero spectral projection of $T$ of the form $r_{0}=T S$ for some $S \in M$. Since $v_{i} r_{0}=\left(p-q_{1}\right) v_{i} r\left(p-q_{1}\right) S$, it follows from Lemma 2.3 that $\left\|P_{\mathcal{F}}\left(v_{i} r_{0}\right)\right\|_{2} \rightarrow 0$ for every subset $\mathcal{F} \subset \Gamma$ that is small relative to $\mathcal{S}$. Replace $p$ by $p-q_{1}, v_{i}$ by $v_{i}\left(p-q_{1}\right), r$ by $r_{0}$ and $B$ by $B\left(p-q_{1}\right)$. We are now in a situation where $B \subset p M p$ is an abelian von Neumann subalgebra normalized by a net of unitaries $\left(v_{i}\right)_{i \in I}$ in $\mathcal{U}(p M p)$ and where $r \in p M p$ is a nonzero projection such that the following properties hold.

- If $t \rightarrow 0$ then $\alpha_{t} \rightarrow$ id in $\|\cdot\|_{2}$-norm uniformly on the tail of $\left(v_{i}\right)_{i \in I}$.

- For every subset $\mathcal{F} \subset \Gamma$ that is small relative to $\mathcal{S}$, we have $\lim _{i}\left\|P_{\mathcal{F}}\left(v_{i} r\right)\right\|_{2}=0$.

- By Proposition 3.9 there exists a sequence of unitaries $w_{n} \in \mathcal{U}(B)$ such that for every $t>0$ we have that $\left\|E_{M}\left(\alpha_{t}\left(w_{n}\right)\right)\right\|_{2} \rightarrow 0$ as $n \rightarrow \infty$.

We shall derive a contradiction from this list of three properties. We separately consider two cases.

Case 1. For every $\varepsilon>0$ and every $b \in \mathcal{U}(B)$ there exists a subset $\mathcal{F} \subset \Gamma$ that is small relative to $\mathcal{S}$ such that

$$
\liminf _{i}\left\|\left(1-P_{\mathcal{F}}\right)\left(v_{i} b v_{i}^{*}\right)\right\|_{2}<\varepsilon
$$

Case 2. There exists a $\delta>0$ and a unitary $b \in \mathcal{U}(B)$ such that for every subset $\mathcal{F} \subset \Gamma$ that is small relative to $\mathcal{S}$, we have

$$
\underset{i}{\limsup }\left\|P_{\mathcal{F}}\left(v_{i} b v_{i}^{*}\right)\right\|_{2} \leq(1-\delta)\|p\|_{2}
$$

First assume that we are in case 1. Denote $\delta_{t}(b)=\alpha_{t}(b)-E_{M}\left(\alpha_{t}(b)\right)$. We claim that $\delta_{t} \rightarrow 0$ in $\|\cdot\|_{2}$-norm uniformly on $r \mathcal{U}(B) r$. To prove this statement, choose $\varepsilon>0$. Put $\delta=\varepsilon^{2} /(9 \tau(p))$ and take $t>0$ small enough and $i_{0} \in I$ such that

$$
\left\|r-\alpha_{t}(r)\right\|_{2} \leq \delta\|p\|_{2} \quad \text { and } \quad\left\|v_{i}-\alpha_{t}\left(v_{i}\right)\right\|_{2} \leq \delta\|p\|_{2} \quad \text { for all } i \geq i_{0} .
$$

We show that $\left\|\delta_{t}(r b r)\right\|_{2} \leq \varepsilon$ for all $b \in \mathcal{U}(B)$, hence proving the claim above. 
Note that our choice of $t$ implies that for all $b \in \mathcal{U}(B)$

$$
\begin{aligned}
& \left\|r \alpha_{t}(b) r-\alpha_{t}(r b r)\right\|_{2} \leq 2 \delta\|p\|_{2} \quad \text { so that } \quad\left\|r \delta_{t}(b) r-\delta_{t}(r b r)\right\|_{2} \leq 4 \delta\|p\|_{2} \quad \text { and } \\
& \left\|v_{i} \alpha_{t}(b) v_{i}^{*}-\alpha_{t}\left(v_{i} b v_{i}^{*}\right)\right\|_{2} \leq 2 \delta\|p\|_{2} \quad \text { for all } i \geq I_{0} .
\end{aligned}
$$

Fix $b \in \mathcal{U}(B)$. Take a subset $\mathcal{F} \subset \Gamma$ that is small relative to $\mathcal{S}$ and such that

$$
\liminf _{i}\left\|\left(1-P_{\mathcal{F}}\right)\left(v_{i} b v_{i}^{*}\right)\right\|_{2}<\delta\|p\|_{2} .
$$

It follows that for all $i \geq i_{0}$,

$$
\begin{aligned}
\left\|\delta_{t}(r b r)\right\|_{2}^{2} & =\left\langle\delta_{t}(r b r), \alpha_{t}(r b r)\right\rangle \leq\left|\left\langle r \delta_{t}(b) r, r \alpha_{t}(b) r\right\rangle\right|+6 \delta \tau(p) \\
& =\left|\left\langle r \delta_{t}(b) r, \alpha_{t}(b)\right\rangle\right|+6 \delta \tau(p)=\left|\left\langle v_{i} r \delta_{t}(b) r v_{i}^{*}, v_{i} \alpha_{t}(b) v_{i}^{*}\right\rangle\right|+6 \delta \tau(p) \\
& \leq\left|\left\langle v_{i} r \delta_{t}(b) r v_{i}^{*}, \alpha_{t}\left(v_{i} b v_{i}^{*}\right)\right\rangle\right|+8 \delta \tau(p) \\
& \leq\left|\left\langle v_{i} r \delta_{t}(b) r v_{i}^{*}, \alpha_{t}\left(P_{\mathcal{F}}\left(v_{i} b v_{i}^{*}\right)\right)\right\rangle\right|+\|p\|_{2}\left\|\left(1-P_{\mathcal{F}}\right)\left(v_{i} b v_{i}^{*}\right)\right\|_{2}+8 \delta \tau(p) .
\end{aligned}
$$

Taking the liminf and using Lemma 4.3 it follows that $\left\|\delta_{t}(r b r)\right\|_{2}^{2} \leq 9 \delta \tau(p)=\varepsilon^{2}$, hence proving the claim above.

Denote by $q$ the smallest projection in $\mathcal{Z}(Q)$ that satisfies $r \leq q$. We conclude from the claim above and from Proposition 3.4 that $\alpha_{t} \rightarrow$ id uniformly on the unit ball of $B q$. This is a contradiction with the existence of the sequence $w_{n} \in \mathcal{U}(B)$ such that $\left\|E_{M}\left(\alpha_{t}\left(w_{n}\right)\right)\right\|_{2} \rightarrow 0$ for every $t>0$.

Next assume that we are in case 2. Take $\delta>0$ and $b \in \mathcal{U}(B)$ such that (4.1) holds. Write $\varepsilon=\delta / 5$ and put $b_{i}:=v_{i} b v_{i}^{*}$. Note that $\alpha_{t} \rightarrow$ id in $\|\cdot\|_{2}$-norm uniformly on the tail of $\left(b_{i}\right)_{i \in I}$. Take $t>0$ and $i_{0} \in I$ such that $\left\|b_{i}-\alpha_{t}\left(b_{i}\right)\right\|_{2} \leq \varepsilon\|p\|_{2}$ for all $i \geq i_{0}$. We claim that $\left\|\delta_{t}(d)\right\|_{2}^{2} \leq(1-\varepsilon) \tau(p)$ for all $d \in \mathcal{U}(B)$. To prove this claim fix $d \in \mathcal{U}(B)$. Take a finite-dimensional subspace $K \subset D \ominus \mathbb{C} 1$ such that, using the notation of Lemma 3.8, we have $\left\|\left(1-Q_{K}\right) \delta_{t}(d)\right\|_{2} \leq \varepsilon\|p\|_{2}$. By Lemma 3.8 take a subset $\mathcal{F} \subset \Gamma$ that is small relative to $\mathcal{S}$ and such that $\left\|Q_{K}\left(b \alpha_{t}(d)\right)\right\|_{2} \leq\left\|P_{\mathcal{F}}(b)\right\|_{2}+\varepsilon\|p\|_{2}$ for all $b$ in the unit ball of $M$.

By our choice of $t$ we know that $\left\|b_{i} \alpha_{t}(d) b_{i}^{*}-\alpha_{t}\left(b_{i} d b_{i}^{*}\right)\right\|_{2} \leq 2 \varepsilon\|p\|_{2}$ for all $i \geq i_{0}$. Since $B$ is abelian, also $d=b_{i} d b_{i}^{*}$. Using that $Q_{K}$ is right $M$-modular it follows that for all $i \geq i_{0}$ we have

$$
\begin{aligned}
\left\|\delta_{t}(d)\right\|_{2}^{2} & =\left\langle\alpha_{t}(d), \delta_{t}(d)\right\rangle=\left\langle\alpha_{t}\left(b_{i} d b_{i}^{*}\right), \delta_{t}(d)\right\rangle \leq\left|\left\langle b_{i} \alpha_{t}(d) b_{i}^{*}, \delta_{t}(d)\right\rangle\right|+2 \varepsilon \tau(p) \\
& \leq\left|\left\langle b_{i} \alpha_{t}(d) b_{i}^{*}, Q_{K}\left(\delta_{t}(d)\right)\right\rangle\right|+3 \varepsilon \tau(p)=\left|\left\langle Q_{K}\left(b_{i} \alpha_{t}(d) b_{i}^{*}\right), \delta_{t}(d)\right\rangle\right|+3 \varepsilon \tau(p) \\
& =\left|\left\langle Q_{K}\left(b_{i} \alpha_{t}(d)\right) b_{i}^{*}, \delta_{t}(d)\right\rangle\right|+3 \varepsilon \tau(p) \leq\left\|Q_{K}\left(b_{i} \alpha_{t}(d)\right)\right\|_{2}\|p\|_{2}+3 \varepsilon \tau(p) \\
& \leq\left\|P_{\mathcal{F}}\left(b_{i}\right)\right\|_{2}\|p\|_{2}+4 \varepsilon \tau(p) .
\end{aligned}
$$

Taking the limsup it follows that $\left\|\delta_{t}(d)\right\|_{2}^{2} \leq(1-\delta+4 \varepsilon) \tau(p)=(1-\varepsilon) \tau(p)$, hence proving the claim.

From this claim, it follows that $\left\|E_{M}\left(\alpha_{t}(d)\right)\right\|_{2}^{2} \geq \varepsilon \tau(p)$ for all $d \in \mathcal{U}(B)$. This is a contradiction with the existence of the sequence $\left(w_{n}\right)$ in $\mathcal{U}(B)$ such that $\left\|E_{M}\left(\alpha_{t}\left(w_{n}\right)\right)\right\|_{2} \rightarrow 0$. This ends the proof of case 2 and also ends the proof of Theorem 4.1 .

\section{Transfer of rigidity}

We fix a trace preserving action $\Gamma \curvearrowright(N, \tau)$ and put $M=N \rtimes \Gamma$. Let $\mathcal{S}$ be a family of subgroups of $\Gamma$. As above we call a subset $\mathcal{F} \subset \Gamma$ small relative to $\mathcal{S}$ if $\mathcal{F}$ can be written as a finite union 
of subsets of the form $g \Sigma h, g, h \in \Gamma, \Sigma \in \mathcal{S}$. Whenever $\mathcal{F} \subset \Gamma$ we denote by $P_{\mathcal{F}}$ the orthogonal projection of $\mathrm{L}^{2}(M)$ onto the closed linear span of $\left\{a u_{g} \mid a \in N, g \in \mathcal{F}\right\}$.

Let $f: \Gamma \rightarrow \mathbb{R}$ be a conditionally negative type function with $f(e)=0$. Define the semigroup $\left(\varphi_{t}\right)_{t>0}$ of unital trace preserving completely positive maps

$$
\varphi_{t}: M \rightarrow M: \varphi_{t}\left(a u_{g}\right)=\exp (-t f(g)) a u_{g} \quad \text { for all } a \in N, g \in \Gamma .
$$

Proposition 5.1. Let $p \in M$ be a projection and assume that $p M p=B \rtimes \Lambda$ is any crossed product decomposition with corresponding canonical unitaries $\left(v_{s}\right)_{s \in \Lambda}$. Let $\Delta: p M p \rightarrow p M p \bar{\otimes} \mathrm{L} \Lambda$ be the comultiplication given by $\Delta\left(b v_{s}\right)=b v_{s} \otimes v_{s}$ for all $b \in B, s \in \Lambda$. Assume that $\left(w_{i}\right)_{i \in I}$ is a net of unitaries in $\mathcal{U}(p M p)$ and that $q \in(\mathrm{L} \Lambda)^{\prime} \cap p M p$ is a projection satisfying

- if $t \rightarrow 0$ then id $\otimes \varphi_{t} \rightarrow$ id in $\|\cdot\|_{2}$-norm uniformly on the tail of $\left(\Delta\left(w_{i}\right)\right)_{i \in I}$,

- for every subset $\mathcal{F} \subset \Gamma$ that is small relative to $\mathcal{S}$, we have $\lim _{i}\left\|\left(1 \otimes P_{\mathcal{F}}\right)\left(\Delta\left(w_{i}\right)(1 \otimes q)\right)\right\|_{2}=0$.

Then there exists a net of elements $\left(s_{j}\right)_{j \in J}$ in $\Lambda$ such that, writing $v_{j}:=v_{s_{j}}$, the following holds.

- If $t \rightarrow 0$ then $\varphi_{t} \rightarrow$ id in $\|\cdot\|_{2}$-norm uniformly on the tail of $\left(v_{j}\right)_{j \in J}$.

- For every subset $\mathcal{F} \subset \Gamma$ that is small relative to $\mathcal{S}$, we have $\lim _{j}\left\|P_{\mathcal{F}}\left(v_{j} q\right)\right\|_{2}=0$.

Proof. Normalize the trace on $M$ such that $\tau(p)=1$. As such $\Delta$ is trace preserving. Take a decreasing sequence $t_{1}>t_{2}>\cdots$ of strictly positive numbers and an increasing sequence $i_{1} \leq i_{2} \leq$ ... such that

$$
1-\operatorname{Re}(\tau \otimes \tau)\left(\Delta\left(w_{i}\right)^{*}\left(\mathrm{id} \otimes \varphi_{t_{n}}\right) \Delta\left(w_{i}\right)\right) \leq 4^{-n-1} \quad \text { for all } i \geq i_{n}
$$

Define

$$
\mathcal{V}_{n}:=\left\{s \in \Lambda \mid 1-\operatorname{Re} \tau\left(v_{s}^{*} \varphi_{t_{n}}\left(v_{s}\right)\right) \leq 2^{-n-1}\right\} .
$$

Fix $n \in \mathbb{N}$ and $i \geq i_{n}$. Write $w_{i}=\sum_{s \in \Lambda} w_{s}^{i} v_{s}$ with $w_{s}^{i} \in B$. It follows that

$$
\begin{aligned}
4^{-n-1} & \geq 1-\operatorname{Re}(\tau \otimes \tau)\left(\Delta\left(w_{i}\right)^{*}\left(\mathrm{id} \otimes \varphi_{t_{n}}\right) \Delta\left(w_{i}\right)\right) \\
& =\sum_{s \in \Lambda}\left(1-\operatorname{Re} \tau\left(v_{s}^{*} \varphi_{t_{n}}\left(v_{s}\right)\right)\right)\left\|w_{s}^{i}\right\|_{2}^{2} \\
& \geq \sum_{s \in \Lambda-\mathcal{V}_{n}} 2^{-n-1}\left\|w_{s}^{i}\right\|_{2}^{2} .
\end{aligned}
$$

We conclude that for all $n \in \mathbb{N}$ and all $i \geq i_{n}$,

$$
\sum_{s \in \Lambda-\mathcal{V}_{n}}\left\|w_{s}^{i}\right\|_{2}^{2} \leq 2^{-n-1}
$$

Define $\mathcal{W}_{n}:=\mathcal{V}_{1} \cap \cdots \cap \mathcal{V}_{n}$. It follows that for all $i \geq i_{n}$

$$
\sum_{s \in \Lambda-\mathcal{W}_{n}}\left\|w_{s}^{i}\right\|_{2}^{2} \leq \frac{1}{2} \quad \text { and hence } \quad \sum_{s \in \mathcal{W}_{n}}\left\|w_{s}^{i}\right\|_{2}^{2} \geq \frac{1}{2} .
$$


We claim that for every $\varepsilon>0, n \in \mathbb{N}$ and subset $\mathcal{F} \subset \Gamma$ that is small relative to $\mathcal{S}$, there exists an $s \in \mathcal{W}_{n}$ satisfying $\left\|P_{\mathcal{F}}\left(v_{s} q\right)\right\|_{2}<\varepsilon$. Indeed, if for a given $\varepsilon>0, n \in \mathbb{N}$ and $\mathcal{F} \subset \Gamma$ that is small relative to $\mathcal{S}$, the claim fails, it would follow that for all $i \geq i_{n}$,

$$
\begin{aligned}
\left\|\left(\operatorname{id} \otimes P_{\mathcal{F}}\right)\left(\Delta\left(w_{i}\right)(1 \otimes q)\right)\right\|_{2}^{2} & =\sum_{s \in \Lambda}\left\|w_{s}^{i}\right\|_{2}^{2}\left\|P_{\mathcal{F}}\left(v_{s} q\right)\right\|_{2}^{2} \\
& \geq \sum_{s \in \mathcal{W}_{n}}\left\|w_{s}^{i}\right\|_{2}^{2}\left\|P_{\mathcal{F}}\left(v_{s} q\right)\right\|_{2}^{2} \geq \sum_{s \in \mathcal{W}_{n}}\left\|w_{s}^{i}\right\|_{2}^{2} \varepsilon^{2} \geq \frac{\varepsilon^{2}}{2} .
\end{aligned}
$$

Since $\lim _{i}\left\|\left(\mathrm{id} \otimes P_{\mathcal{F}}\right)\left(\Delta\left(w_{i}\right)(1 \otimes q)\right)\right\|_{2}=0$ this is absurd and the claim is proven.

For every $\varepsilon>0, n \in \mathbb{N}$ and $\mathcal{F} \subset \Gamma$ small relative to $\mathcal{S}$, pick an element $s_{\varepsilon, n, \mathcal{F}} \in \mathcal{W}_{n}$ such that $\left\|P_{\mathcal{F}}\left(v_{s_{\varepsilon, n, \mathcal{F}}} q\right)\right\|_{2}<\varepsilon$. We obtain a net $\left(s_{j}\right)_{j \in J}$ in $\Lambda$ that satisfies all the conclusions of the proposition. Indeed, first observe that for every fixed $s \in \Lambda$ the expression $\tau\left(v_{s}^{*} \varphi_{t}\left(v_{s}\right)\right)$ increases when $t>0$ decreases. Writing $v_{\varepsilon, n, \mathcal{F}}=v_{s_{\varepsilon, n, \mathcal{F}}}$ and using the inequality $\left\|\varphi_{t}\left(v_{s}\right)-v_{s}\right\|_{2}^{2} \leq 2\left(1-\operatorname{Re} \tau\left(v_{s}^{*} \varphi_{t}\left(v_{s}\right)\right)\right)$, it follows that

$$
\begin{array}{cl}
\left\|\varphi_{t}\left(v_{\varepsilon, n, \mathcal{F}}\right)-v_{\varepsilon, n, \mathcal{F}}\right\|_{2} \leq 2^{-n_{0} / 2} & \text { whenever } 0<t \leq t_{n_{0}} \text { and } n \geq n_{0} \\
\left\|P_{\mathcal{F}_{0}}\left(v_{\varepsilon, n, \mathcal{F}} q\right)\right\|_{2}<\varepsilon & \text { whenever } \quad \mathcal{F}_{0} \subset \mathcal{F}
\end{array}
$$

\section{Proof of Theorems 1.2 and 1.3}

We are given a crossed product $\mathrm{II}_{1}$ factor $M=N \rtimes \Gamma$. Fix a projection $p \in M$ and assume that $p M p=B \rtimes \Lambda$ is another crossed product decomposition with $B$ being diffuse and of type I. Denote by $\left(v_{s}\right)_{s \in \Lambda}$ the canonical unitaries in $B \rtimes \Lambda$. Since $M$ is a factor, the action $\Lambda \curvearrowright \mathcal{Z}(B)$ is ergodic and hence $B \cong \mathrm{M}_{m}(\mathbb{C}) \otimes \mathcal{Z}(B)$ for some integer $m$.

Denote by $\Delta: p M p \rightarrow p M p \bar{\otimes} \mathrm{L} \Lambda$ the comultiplication given by $\Delta\left(b v_{s}\right)=b v_{s} \otimes v_{s}$ for all $b \in B$, $s \in \Lambda$

Since $M$ is a $\mathrm{II}_{1}$ factor and $B \subset p M p$ is diffuse, we can take partial isometries $V_{1}, \ldots, V_{k} \in M$ such that $V_{1}=p, V_{i}^{*} V_{i} \in B$ for all $i=1, \ldots, k$ and $\sum_{i} V_{i} V_{i}^{*}=1$. We extend $\Delta$ to a unital *-homomorphism $M \rightarrow M \bar{\otimes} \mathrm{L} \Lambda$ by the formula

$$
\Delta(x):=\sum_{i, j=1}^{k}\left(V_{i} \otimes 1\right) \Delta\left(V_{i}^{*} x V_{j}\right)\left(V_{j}^{*} \otimes 1\right) .
$$

Since $V_{1}=p$, the restriction of the new $\Delta$ to $p M p$ equals the original comultiplication.

The following meta-theorem brings together all that we have done in the previous sections. Our main Theorem 1.2 will be a direct consequence.

Theorem 6.1. Within the setup described before the theorem, let $b$ be an unbounded 1-cocycle into the orthogonal representation $\pi: \Gamma \rightarrow \mathcal{O}\left(H_{\mathbb{R}}\right)$ that is mixing relative to a family $\mathcal{S}$ of subgroups of $\Gamma$ such that $b$ is bounded on every $\Sigma \in \mathcal{S}$. Define $\widetilde{M}$ and $\left(\alpha_{t}\right)_{t \in \mathbb{R}}$ as in paragraph 3.1. Assume that $Q \subset M$ is a diffuse von Neumann subalgebra and that $q \in(\mathrm{L} \Lambda)^{\prime} \cap p M p$ is a nonzero projection such that the following two conditions hold. 
1. id $\otimes \alpha_{t} \rightarrow$ id in $\|\cdot\|_{2}$-norm uniformly on the unit ball of $\Delta(Q)$.

2. For every $\Sigma \in \mathcal{S}$ we have $\Delta(Q)(1 \otimes q) \nprec M \bar{\otimes}(N \rtimes \Sigma)$.

Then there exists $\Sigma \in \mathcal{S}$ such that $B \prec N \rtimes \Sigma$ and hence $(B)_{1} \subset_{\text {approx }} N \rtimes \mathcal{S}$.

Proof. Since $Q$ is diffuse, we may, after a unitary conjugacy of $Q$, assume that $p \in Q$. It follows that $\Delta(p Q p)(1 \otimes q) \nprec p M p \bar{\otimes}(N \rtimes \Sigma)$ for all $\Sigma \in \mathcal{S}$. Lemma 2.4 provides a net of unitaries $\left(w_{i}\right)$ in $\mathcal{U}(p Q p)$ such that $\left\|\left(1 \otimes P_{\mathcal{F}}\right)\left(\Delta\left(w_{i}\right)(1 \otimes q)\right)\right\|_{2} \rightarrow 0$ for every subset $\mathcal{F} \subset \Gamma$ that is small relative to $\mathcal{S}$. Since id $\otimes \alpha_{t} \rightarrow$ id in $\|\cdot\|_{2}$-norm uniformly on the unit ball of $\Delta(Q)$, certainly id $\otimes \alpha_{t} \rightarrow$ id in $\|\cdot\|_{2}$-norm uniformly on the unitaries $\Delta\left(w_{i}\right) \in \mathcal{U}(p M p \bar{\otimes} p M p)$. By the transfer of rigidity proposition 5.1 we find a net of elements $\left(s_{j}\right)$ in $\Lambda$ such that, writing $v_{j}:=v_{s_{j}}$, we have that

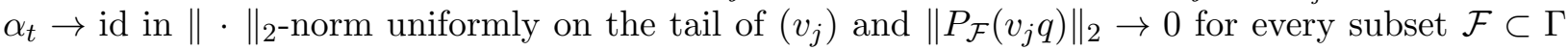
that is small relative to $\mathcal{S}$.

Since $\mathcal{Z}(B)$ is an abelian von Neumann subalgebra of $p M p$ that is normalized by the unitaries $v_{j}$ and that is moreover regular in $p M p$, it follows from Theorem 4.1 and Lemma 3.3 that $\alpha_{t} \rightarrow$ id in $\|\cdot\|_{2^{-}}$ norm uniformly on the unit ball of $\mathcal{Z}(B)$, and hence as well on the unit ball of $B=\mathrm{M}_{m}(\mathbb{C}) \otimes \mathcal{Z}(B)$. If for every $\Sigma \in \mathcal{S}$ we would have that $B \nprec N \rtimes \Sigma$, Theorem 3.10 would imply that $\alpha_{t} \rightarrow$ id in $\|\cdot\|_{2}$-norm uniformly on the unit ball of $p M p$. This would be a contradiction with $b$ being unbounded.

So there exists a $\Sigma \in \mathcal{S}$ such that $B \prec N \rtimes \Sigma$. Since $B \subset p M p$ is regular Proposition 2.6 implies that $(B)_{1} \subset$ approx $N \rtimes \mathcal{S}$.

In order to establish the condition $\Delta(Q)(1 \otimes q) \nprec M \bar{\otimes}(N \rtimes \Sigma)$ appearing in Theorem 6.1, we prove the following lemma. It is contained in [IPV10, Lemma 9.2] and [HPV10, Lemma 4] but we include a proof for the convenience of the reader.

We say that a finite von Neumann algebra $P$ is anti-(T) if there exists a chain of von Neumann subalgebras $\mathbb{C} 1=P_{0} \subset P_{1} \subset \cdots \subset P_{n}=P$ such that for every $i=1, \ldots, n$ the finite von Neumann algebra $P_{i}$ has property $(\mathrm{H})$ relative to $P_{i-1}$ in the sense of [Po01, Section 2]. Examples include crossed products $A \rtimes \Sigma$ where $A$ is amenable and $\Sigma$ admits a chain of subgroups $\{e\}=\Sigma_{0}<\Sigma_{1}<$ $\cdots<\Sigma_{n}=\Sigma$ such that for all $i \in\{1, \ldots, n\}$ the subgroup $\Sigma_{i-1} \triangleleft \Sigma_{i}$ is normal and the quotient group $\Sigma_{i} / \Sigma_{i-1}$ has the Haagerup property.

Lemma 6.2. If the bimodule ${ }_{p M p} \mathcal{H}_{p M p}$ is weakly contained in the coarse $p M p$-pMp-bimodule then the bimodule ${ }_{\Delta(M)}\left(\mathrm{L}^{2}(M) \otimes \mathcal{H}\right)_{\Delta(M)}$ is weakly contained in the coarse $M$-M-bimodule.

Let $Q, P \subset M$ be von Neumann subalgebras.

- If $Q$ has no amenable direct summand and $P$ is amenable, then $\Delta(Q) \nprec M \bar{\otimes} P$.

- If $Q$ is diffuse with property (T) and $P$ is anti-(T), then $\Delta(Q) \nprec M \bar{\otimes} P$.

Proof. Put $\mathcal{M}=B \rtimes \Lambda=p M p$. Denote by $\sigma: \mathcal{M} \bar{\otimes} \mathcal{M} \rightarrow \mathcal{M} \bar{\otimes} \mathcal{M}$ the flip automorphism. We first claim that the bimodule

$$
\Delta(\mathcal{M}) \otimes 1 \mathrm{~L}^{2}(\mathcal{M} \bar{\otimes} \mathcal{M} \bar{\otimes} \mathcal{M})_{(\mathrm{id} \otimes \sigma)(\Delta(\mathcal{M}) \otimes 1)}
$$

is weakly contained in the coarse $\mathcal{M}$ - $\mathcal{M}$-bimodule. To prove this claim, observe that

$$
\Delta(\mathcal{M}) \otimes 1\left(\mathrm{~L}^{2}(\mathcal{M}) \otimes \ell^{2}(\Lambda) \otimes \mathrm{L}^{2}(\mathcal{M})\right)_{\mathcal{M} \bar{\otimes} 1 \bar{\otimes} \mathcal{M}}
$$


is unitarily isomorphic with the tensor product $\mathrm{L}^{2}(\mathcal{M}) \otimes_{B} \mathrm{~L}^{2}(\mathcal{M} \bar{\otimes} \mathcal{M})$ of the bimodules $\mathcal{M}^{2}(\mathcal{M})_{B}$ and ${ }_{B \otimes 1} \mathrm{~L}^{2}(\mathcal{M} \bar{\otimes} \mathcal{M})_{\mathcal{M}} \bar{\otimes} \mathcal{M}$. Since $B$ is amenable this relative tensor product is weakly contained in the coarse bimodule. Restricting the right $(\mathcal{M} \bar{\otimes} \mathcal{M})$-module action to $\Delta(\mathcal{M})$, the claim follows. We extended $\Delta$ from $\mathcal{M}$ to $M$. In the bimodule picture this amounts to tensoring on the left by $\mathrm{L}^{2}(M p)$ and on the right by $\mathrm{L}^{2}(p M)$. It follows that

$$
\Delta(M) \otimes{ }_{1} \mathrm{~L}^{2}(M \bar{\otimes} \mathcal{M} \bar{\otimes} \mathcal{M})_{(\mathrm{id} \otimes \sigma)(\Delta(M) \otimes 1)}
$$

is weakly contained in the coarse $M$ - $M$-bimodule. From this the first statement of the lemma follows immediately.

Assume that $P$ is amenable and that $\Delta(Q) \prec M \bar{\otimes} P$. We prove that $Q$ has an amenable direct summand. Since $P$ is amenable, the coarse $P$ - $P$-bimodule $P \otimes{ }_{1} \mathrm{~L}^{2}(P \bar{\otimes} P)_{1 \otimes P}$ contains a sequence of vectors $\xi_{n}$ satisfying the following properties.

$$
\left\|(a \otimes 1) \xi_{n}-\xi_{n}(1 \otimes a)\right\|_{2} \rightarrow 0 \quad \text { and } \quad\left\langle(a \otimes 1) \xi_{n}, \xi_{n}\right\rangle \rightarrow \tau(a) \text { for all } a \in P .
$$

View $\mathrm{L}^{2}(P \bar{\otimes} P) \subset \mathrm{L}^{2}(M \bar{\otimes} M)$ and identify $\mathrm{L}^{2}(M \bar{\otimes} M)$ with the space of Hilbert Schmidt operators on $\mathrm{L}^{2}(M)$. Since $\xi_{n} \in \mathrm{L}^{2}(P \bar{\otimes} P)$ we have for all $a \in M$ that

$$
\begin{aligned}
\left\langle(a \otimes 1) \xi_{n}, \xi_{n}\right\rangle & =(\tau \otimes \tau)\left((a \otimes 1) \xi_{n} \xi_{n}^{*}\right)=(\tau \otimes \tau)\left(\left(E_{P}(a) \otimes 1\right) \xi_{n} \xi_{n}^{*}\right) \\
& =\left\langle\left(E_{P}(a) \otimes 1\right) \xi_{n}, \xi_{n}\right\rangle \rightarrow \tau\left(E_{P}(a)\right)=\tau(a) .
\end{aligned}
$$

So every $\xi_{n}$ gives rise to a Hilbert Schmidt operator $S_{n}$ on $\mathrm{L}^{2}(M)$ and hence a trace class operator $T_{n}:=S_{n} S_{n}^{*} \in \mathcal{T C}\left(\mathrm{L}^{2}(M)\right)^{+}$. By construction, $T_{n}$ has the following properties

$$
\left\|a T_{n}-T_{n} a\right\|_{1, \operatorname{Tr}} \rightarrow 0 \text { for all } a \in P \quad \text { and } \operatorname{Tr}\left(b T_{n}\right) \rightarrow \tau(b) \text { for all } b \in M .
$$

Since $\Delta(Q) \prec M \bar{\otimes} P$, take a nonzero partial isometry $v \in \mathrm{M}_{1, k}(\mathbb{C}) \bar{\otimes} M \bar{\otimes} p M$ and a, possibly non-unital, $*$-homomorphism $\theta: Q \rightarrow \mathrm{M}_{k}(\mathbb{C}) \bar{\otimes} M \bar{\otimes} P$ satisfying $\Delta(a) v=v \theta(a)$ for all $a \in Q$. Denote $q:=v v^{*}$ and note that $q \in \Delta(Q)^{\prime} \cap M \bar{\otimes} \mathcal{M}$.

The operator $R_{n}:=v\left(1 \otimes 1 \otimes T_{n}\right) v^{*}$ is a positive element of $M \bar{\otimes} \mathrm{B}\left(p \mathrm{~L}^{2}(M)\right)$ satisfying $(\tau \otimes \operatorname{Tr})\left(R_{n}\right)<$ $\infty$. The square root $\eta_{n}:=R_{n}^{1 / 2}$ can be viewed as a vector in $\mathrm{L}^{2}(M) \otimes p \mathrm{~L}^{2}(M) \otimes \mathrm{L}^{2}(M) p$ and satisfies by construction the following properties.

$$
\begin{aligned}
& \left\|(\Delta(a) \otimes 1) \eta_{n}-\eta_{n}(\mathrm{id} \otimes \sigma)(\Delta(a) \otimes 1)\right\|_{2} \rightarrow 0 \text { for all } a \in Q \text { and } \\
& \left\langle(b \otimes 1) \eta_{n}, \eta_{n}\right\rangle \rightarrow \tau(b q) \text { for all } b \in M \bar{\otimes} \mathcal{M} .
\end{aligned}
$$

Define $z \in \mathcal{Z}(Q)$ such that $\Delta(z)$ is the support projection of $E_{\Delta(Q)}(q)$. We have shown that the bimodule

$$
\Delta(Q) \otimes{ }_{1} \mathrm{~L}^{2}(M \bar{\otimes} \mathcal{M} \bar{\otimes} \mathcal{M})(\mathrm{id} \otimes \sigma)(\Delta(Q) \otimes 1)
$$

weakly contains the trivial $Q z$-bimodule. By the first statement of the lemma the bimodule in 6.1$)$ is weakly contained in the coarse $Q$ - $Q$-bimodule. It follows that $Q z$ is an amenable direct summand of $Q$.

Finally assume that $P$ is anti-(T), that $Q$ is diffuse with property $(\mathrm{T})$ and that $\Delta(Q) \prec M \bar{\otimes} P$. Let $\mathbb{C} 1=P_{0} \subset P_{1} \subset \cdots \subset P_{n}=P$ be a chain of von Neumann subalgebras such that for every $i=1, \ldots, n, P_{i}$ has property $(\mathrm{H})$ relative to $P_{i-1}$. Repeatedly applying [HPV10, Lemma 1] it follows that $\Delta(Q) \prec M \bar{\otimes} P_{i}$ for every $i$ and hence $\Delta(Q) \prec M \bar{\otimes} 1$. By the previous statement of the lemma, $Q$ has an amenable direct summand. This is a contradiction with $Q$ being diffuse with property $(\mathrm{T})$. 


\section{Proof of Theorem 1.2}

We finally prove Theorem 1.2 . We now also require that $N=A$ is of type I. Since $M$ is a factor the action $\Gamma \curvearrowright \mathcal{Z}(A)$ is ergodic and since $A$ is of type $\mathrm{I}$ it follows that $A \cong \mathrm{M}_{n}(\mathbb{C}) \otimes \mathcal{Z}(A)$ for some integer $n$.

The case $\boldsymbol{\Gamma} \in \mathcal{C}$. Let $H<\Gamma$ be a nonamenable subgroup with the relative property $(\mathrm{T})$. Then, $Q=\mathrm{L} H$ satisfies the conditions in Theorem 6.1. Condition 1 follows directly from the relative property $(\mathrm{T})$ of $\Delta(Q)$ inside $M \bar{\otimes} p M p$. Condition 2 follows from Lemma 6.2 and the observation that all the von Neumann algebras $A \rtimes \Sigma, \Sigma \in \mathcal{S}$, are amenable.

The case $\Gamma \in \mathcal{D}$. Let $H<\Gamma$ be an infinite subgroup with the plain property (T). Then, $Q=\mathrm{L} H$ satisfies the conditions in Theorem 6.1. Condition 1 follows directly from the property $(\mathrm{T})$ of $\Delta(Q)$, while condition 2 follows from Lemma 6.2 and the observation that all the von Neumann algebras $A \rtimes \Sigma, \Sigma \in \mathcal{S}$, are anti-(T).

The case $\Gamma \in \mathcal{E}$. Let $H<\Gamma$ be a nonamenable subgroup with a nonamenable centralizer $H^{\prime}<\Gamma$. We claim that $Q=\mathrm{L} H$ satisfies the conditions of Theorem 6.1. Condition 2 follows from Lemma 6.2 and the observation that all the von Neumann algebras $A \rtimes \Sigma, \Sigma \in \mathcal{S}$, are amenable. We now prove condition 1 using a spectral gap argument.

We are given the orthogonal representation $\pi: \Gamma \rightarrow \mathcal{O}\left(H_{\mathbb{R}}\right)$ that is weakly contained in the regular representation and the 1-cocycle $b: \Gamma \rightarrow H_{\mathbb{R}}$. With these data we build the automorphisms $\left(\alpha_{t}\right)$ on $\widetilde{M}$ as in paragraph 3.1. Define $\mathcal{H}:=p \mathrm{~L}^{2}(\widetilde{M} \ominus M) p$. From Lemma 3.5 it follows that ${ }_{p M p} \mathcal{H}_{p M p}$ is weakly contained in the coarse $p M p-p M p$-bimodule. By Lemma 6.2 the bimodule ${ }_{\Delta(M)}\left(\mathrm{L}^{2}(M) \otimes \mathcal{H}\right)_{\Delta(M)}$ is weakly contained in the coarse $M$ - $M$-bimodule. Therefore the unitary representation

$$
\gamma: H^{\prime} \rightarrow \mathcal{U}\left(\mathrm{L}^{2}(M) \otimes \mathcal{H}\right): \gamma(g) \xi:=\Delta\left(u_{g}\right) \xi \Delta\left(u_{g}^{*}\right)
$$

is weakly contained in the regular representation. Since $H^{\prime}$ is nonamenable, $\gamma$ does not weakly contain the trivial $H^{\prime}$-representation.

Choose $\varepsilon>0$. Take $g_{1}, \ldots, g_{n} \in H^{\prime}$ and $\rho>0$ such that every vector $\xi \in \mathrm{L}^{2}(M) \otimes \mathcal{H}$ satisfying $\left\|\xi-\gamma\left(g_{k}\right) \xi\right\| \leq \rho$ for all $k=1, \ldots, n$, must be of norm at most $\varepsilon$, i.e. satisfies $\|\xi\| \leq \varepsilon$. Put $\delta=\min \{\rho / 12, \varepsilon\}$. Take $t>0$ small enough such that

$$
\left\|\alpha_{t}(p)-p\right\|_{2} \leq \delta \quad \text { and } \quad\left\|\left(\mathrm{id} \otimes \alpha_{t}\right) \Delta\left(u_{g_{k}}\right)-\Delta\left(u_{g_{k}}\right)\right\|_{2} \leq \delta \quad \text { for all } k=1, \ldots, n .
$$

We claim that $\left\|\left(\mathrm{id} \otimes \alpha_{t}\right) \Delta(b)-\Delta(b)\right\|_{2} \leq 5 \sqrt{2} \varepsilon$ for all $b$ in the unit ball of $Q$. Once this claim is proven, also condition 1 of Theorem 6.1 has been verified and the theorem follows from Theorem 6.1 .

Fix $b \in(Q)_{1}$. Write $\xi=\left(\right.$ id $\left.\otimes \alpha_{t}\right) \Delta(b), \xi^{\prime}=(1 \otimes p) \xi(1 \otimes p)$ and $\xi^{\prime \prime}=\xi^{\prime}-\left(\right.$ id $\left.\otimes E_{M}\right)\left(\xi^{\prime}\right)$. Note that $\xi^{\prime \prime} \in \mathrm{L}^{2}(M) \otimes \mathcal{H}$. Observe that $\left\|\xi-\xi^{\prime}\right\|_{2} \leq 2 \delta$. Since $b$ commutes with $u_{g_{k}}$, we get that $\left\|\xi-\Delta\left(u_{g_{k}}\right) \xi \Delta\left(u_{g_{k}}^{*}\right)\right\|_{2} \leq 2 \delta$ for all $k=1, \ldots, n$. Hence, $\left\|\xi^{\prime}-\Delta\left(u_{g_{k}}\right) \xi^{\prime} \Delta\left(u_{g_{k}}^{*}\right)\right\|_{2} \leq 6 \delta$ and so $\left\|\xi^{\prime \prime}-\gamma\left(g_{k}\right) \xi^{\prime \prime}\right\|_{2} \leq 12 \delta \leq \rho$. We conclude that $\left\|\xi^{\prime \prime}\right\|_{2} \leq \varepsilon$. Hence, $\left\|\xi-\left(\mathrm{id} \otimes E_{M}\right)(\xi)\right\|_{2} \leq \varepsilon+4 \delta \leq 5 \varepsilon$. This means that $\left\|\left(\mathrm{id} \otimes \delta_{t}\right) \Delta(b)\right\|_{2} \leq 5 \varepsilon$. It follows from Lemma 3.1 that $\left\|\Delta(b)-\left(\mathrm{id} \otimes \alpha_{t}\right) \Delta(b)\right\|_{2} \leq$ $5 \sqrt{2} \varepsilon$, proving the claim above.

The case $\Gamma \in \mathcal{C}_{2}$. We have $\Gamma=\Gamma_{1} \times \Gamma_{2}$ with $\Gamma_{i} \in \mathcal{C}$. So we are given nonamenable subgroups $H_{i}<\Gamma_{i}$ with the relative property $(\mathrm{T})$ and families $\mathcal{S}_{i}$ of subgroups of $\Gamma_{i}$. We can view $M$ as the crossed product $M=\left(A \rtimes \Gamma_{1}\right) \rtimes \Gamma_{2}$ or as the crossed product $\left(A \rtimes \Gamma_{2}\right) \rtimes \Gamma_{1}$. This gives rise to the malleable deformations $\left(\alpha_{t}^{1}\right)$ and $\left(\alpha_{t}^{2}\right)$ associated with the unbounded cocycles $b_{i}: \Gamma_{i} \rightarrow H_{\mathbb{R}}^{i}$ into the orthogonal representations $\pi^{i}: \Gamma_{i} \rightarrow \mathcal{O}\left(H_{\mathbb{R}}^{i}\right)$. 
Denote $Q=\mathrm{L}\left(H_{1} \times H_{2}\right)$. We prove below the existence of a nonzero projection $q \in p M p \cap(\mathrm{L} \Lambda)^{\prime}$ such that for all $\Sigma_{2} \in \mathcal{S}_{2}$, we have

$$
\Delta(Q)(1 \otimes q) \nprec p M p \bar{\otimes}\left(A \rtimes\left(\Gamma_{1} \times \Sigma_{2}\right)\right)
$$

Since $\Delta(Q) \subset M \bar{\otimes} p M p$ has the relative property (T), 6.2 and Theorem 6.1 imply that $B \subset_{\text {approx }}$ $\left(A \rtimes \Gamma_{1}\right) \rtimes \mathcal{S}_{2}$. By symmetry also $B \subset_{\text {approx }}\left(A \rtimes \Gamma_{2}\right) \rtimes \mathcal{S}_{1}$. By Lemma 2.7 it follows that there exist $\Sigma_{i} \in \mathcal{S}_{i}$ such that $B \prec A \rtimes\left(\Sigma_{1} \times \Sigma_{2}\right)$, ending the proof of the case $\Gamma \in \mathcal{C}_{2}$. It remains to settle (6.2).

Denote by Ball $N$ the unit ball of a von Neumann algebra $N$. Let $p_{1} \in \Delta\left(\mathrm{L}\left(H_{1} \times H_{2}\right)\right)^{\prime} \cap M \bar{\otimes} p M p$ be as in Proposition 2.6 the maximal projection such that

$$
\operatorname{Ball}\left(\Delta\left(\mathrm{L}\left(H_{1} \times H_{2}\right)\right) p_{1}\right) \subset_{\text {approx }} M \bar{\otimes}\left(A \rtimes\left(\Gamma_{1} \times \mathcal{S}_{2}\right)\right) .
$$

Define $f_{i}$ as the smallest projection in $\Delta\left(\mathrm{L}\left(\Gamma_{i}\right)\right)^{\prime} \cap M \bar{\otimes} p M p$ that satisfies $p_{1} \leq f_{i}$. Define $p_{2}$ as the smallest projection in $\Delta(\mathrm{L} \Gamma)^{\prime} \cap M \bar{\otimes} p M p$ that satisfies $p_{1} \leq p_{2}$. Note that $f_{i} \leq p_{2}$ for both $i=1,2$.

Since $\operatorname{Ball}\left(\Delta\left(\mathrm{L}\left(H_{2}\right)\right) p_{1}\right) \subset_{\text {approx }} M \bar{\otimes}\left(A \rtimes\left(\Gamma_{1} \times \mathcal{S}_{2}\right)\right)$ and since $H_{2}$ is nonamenable, Lemmas 2.7 and 6.2 imply that $\Delta\left(\mathrm{L}\left(H_{2}\right)\right) p_{1} \nprec M \bar{\otimes}\left(A \rtimes\left(\mathcal{S}_{1} \times \Gamma_{2}\right)\right)$. Since $H_{2}<\Gamma_{2}$ has the relative property (T), we know that id $\otimes \alpha_{t}^{1} \rightarrow$ id in $\|\cdot\|_{2}$-norm uniformly on the unit ball of $\Delta\left(\mathrm{L}\left(H_{2}\right)\right) p_{1}$. Both statements, together with Theorem 3.10 and the observation that $\mathrm{L}\left(\Gamma_{1}\right)$ commutes with $\mathrm{L}\left(H_{2}\right)$, imply that id $\otimes \alpha_{t}^{1} \rightarrow$ id in $\|\cdot\|_{2}$-norm uniformly on the unit ball of $\Delta\left(\mathrm{L}\left(\Gamma_{1}\right)\right) f_{1}$. Since the normalizer of $\mathrm{L}\left(\Gamma_{1}\right)$ contains L $(\Gamma)$, Lemma 3.3 implies that id $\otimes \alpha_{t}^{1} \rightarrow$ id in $\|\cdot\|_{2}$-norm uniformly on the unit ball of $\Delta\left(\mathrm{L}\left(\Gamma_{1}\right)\right) p_{2}$. Using $H_{1}$ instead of $H_{2}$, we also find that id $\otimes \alpha_{t}^{1} \rightarrow$ id in $\|\cdot\|_{2}$-norm uniformly on the unit ball of $\Delta\left(\mathrm{L}\left(\Gamma_{2}\right)\right) p_{2}$. The unitaries $\Delta\left(u_{(g, h)}\right)=\Delta\left(u_{(g, e)}\right) \Delta\left(u_{(e, h)}\right)$ form a group generating $\Delta(\mathrm{L} \Gamma)$. It follows from Proposition 3.4 that id $\otimes \alpha_{t}^{1} \rightarrow$ id in $\|\cdot\|_{2}$-norm uniformly on the unit ball of $\Delta(\mathrm{L} \Gamma) p_{2}$.

Define $p_{3}$ as the smallest projection in $\Delta(M)^{\prime} \cap M \bar{\otimes} p M p$ that satisfies $p_{1} \leq p_{3}$. Note that $p_{2} \leq p_{3}$. We observed above that $\Delta\left(\mathrm{L}\left(H_{2}\right)\right) p_{1} \nprec M \bar{\otimes}\left(A \rtimes\left(\mathcal{S}_{1} \times \Gamma_{2}\right)\right)$. The relative property (T) of $\Delta\left(\mathrm{L}\left(H_{2}\right)\right)$ in $M \bar{\otimes} p M p$ implies that id $\otimes \alpha_{t}^{1} \rightarrow$ id in $\|\cdot\|_{2}$-norm uniformly on the unit ball of $\Delta\left(\mathrm{L}\left(H_{2}\right)\right)$. By Lemma 2.4 take a net of elements $g_{i} \in H_{2}$ such that $\left\|\left(1 \otimes P_{\mathcal{F} \times \Gamma_{2}}\right)\left(\Delta\left(u_{g_{i}}\right) p_{1}\right)\right\|_{2} \rightarrow 0$ for every subset $\mathcal{F} \subset \Gamma_{1}$ that is small relative to $\mathcal{S}_{1}$. Since the unitaries $\Delta\left(u_{g_{i}}\right)$ normalize the abelian von Neumann algebra $\Delta(\mathcal{Z}(A))$ and since $\mathcal{Z}(A) \subset M$ is regular, it follows from Theorem 4.1 that id $\otimes \alpha_{t}^{1} \rightarrow$ id in $\|\cdot\|_{2}$-norm uniformly on the unit ball of $\Delta(\mathcal{Z}(A)) p_{3}$ and hence also on the unit ball of $\Delta(A) p_{3}$. The group of unitaries $\Delta\left(a u_{g}\right), a \in \mathcal{U}(A), g \in \Gamma$, generates $\Delta(M)$. So, the uniform convergence id $\otimes \alpha_{t}^{1} \rightarrow$ id on the unit balls of $\Delta(\mathrm{L} \Gamma) p_{2}$ and $\Delta(A) p_{3}$, together with Proposition 3.4 , implies that id $\otimes \alpha_{t}^{1} \rightarrow$ id in $\|\cdot\|_{2}$-norm uniformly on the unit ball of $\Delta(M) p_{3}$.

Recall how $\Delta$ was extended to $M$ starting from the comultiplication on $p M p$. Put $S_{i}:=V_{i} V_{i}^{*}$ and $P_{i}:=V_{i}^{*} V_{i} \in B$. By construction $\Delta\left(S_{i}\right)=S_{i} \otimes 1$ and hence $p_{3}$ commutes with all the projections $S_{i} \otimes 1$. Define $p_{i}:=\left(V_{i}^{*} \otimes 1\right) p_{3}\left(V_{i} \otimes 1\right)$. For all $b \in \mathcal{U}(B)$ and $s, r \in \Lambda$, we have

$$
p_{i}\left(b v_{s} \otimes v_{r}\right)=\left(V_{i}^{*} \otimes 1\right) p_{3} \Delta\left(V_{i} b v_{r}\right)\left(v_{r^{-1} s} \otimes 1\right) .
$$

We know that id $\otimes \alpha_{t}^{1} \rightarrow$ id in $\|\cdot\|_{2}$-norm uniformly on the unit ball of $p_{3} \Delta(M)$. So, id $\otimes \alpha_{t}^{1} \rightarrow$ id in $\|\cdot\|_{2}$-norm uniformly on the elements $\Delta\left(V_{i} b v_{r}\right), b \in \mathcal{U}(B), r \in \Lambda$. Formula (6.3) then implies that $\mathrm{id} \otimes \alpha_{t}^{1} \rightarrow \mathrm{id}$ in $\|\cdot\|_{2}$-norm uniformly on the elements $p_{i}\left(b v_{s} \otimes v_{r}\right), b \in \mathcal{U}(B), s, r \in \Lambda$. As in Lemma 3.3 let $q_{1} \in(\mathrm{L} \Lambda)^{\prime} \cap p M p$ be the maximal projection such that $\alpha_{t}^{1} \rightarrow$ id in $\|\cdot\|_{2}$-norm uniformly on the unit ball of $\mathrm{L}(\Lambda) q_{1}$. Since the unitaries $b v_{s} \otimes v_{r}$ form a group generating $p M p \bar{\otimes} \mathrm{L} \Lambda$ and since id $\otimes \alpha_{t}^{1} \rightarrow$ id in $\|\cdot\|_{2}$-norm uniformly on the elements $p_{i}\left(b v_{s} \otimes v_{r}\right)$, it follows from Proposition 3.4 
that $p_{i} \leq p \otimes q_{1}$ for every $i=1, \ldots, k$. So $p_{3} \leq 1 \otimes q_{1}$ and in particular $p_{1} \leq 1 \otimes q_{1}$. If $q_{1}<p$, we can put $q=p-q_{1}$ and 6.2 is proven.

As a final step, we assume that $q_{1}=p$ and derive a contradiction. So, $\alpha_{t}^{1} \rightarrow$ id in $\|\cdot\|_{2}$-norm uniformly on the unit ball of $\mathrm{L}(\Lambda)$. We observed above that $\Delta\left(\mathrm{L}\left(H_{2}\right)\right) p_{1} \nprec M \bar{\otimes}\left(A \rtimes\left(\mathcal{S}_{1} \times \Gamma_{2}\right)\right)$. Since $\Delta(M) \subset M \bar{\otimes} \mathrm{L} \Lambda$, it is then impossible that

$$
\operatorname{Ball}(M \bar{\otimes} \mathrm{L}(\Lambda)) \subset_{\text {approx }} M \bar{\otimes}\left(A \rtimes\left(\mathcal{S}_{1} \times \Gamma_{2}\right)\right) .
$$

By Lemma 2.4 and Proposition 2.6 we find a nonzero projection $e \in(\mathrm{L} \Lambda)^{\prime} \cap p M p$ and a net of elements $\left(s_{j}\right)$ in $\Lambda$ such that $\left\|P_{\mathcal{F} \times \Gamma_{2}}\left(v_{s_{j}} e\right)\right\|_{2} \rightarrow 0$ for every subset $\mathcal{F} \subset \Gamma_{1}$ that is small relative to $\mathcal{S}_{1}$. Since the unitaries $v_{s_{j}}$ normalize the regular abelian von Neumann subalgebra $\mathcal{Z}(B) \subset p M p$, it follows from Theorem 4.1 that $\alpha_{t}^{1} \rightarrow$ id in $\|\cdot\|_{2}$-norm uniformly on the unit ball of $\mathcal{Z}(B)$. Together with the uniform convergence on the unit ball of $\mathrm{L}(\Lambda)$ and Proposition 3.4 , we obtain the uniform convergence on the unit ball of $p M p$. This is absurd because the cocycle $b_{1}$ is unbounded.

The case $\Gamma \in \mathcal{D}_{2}$. The proof is identical to the proof of the case $\Gamma \in \mathcal{C}_{2}$, but now using the last statement of Lemma 6.2.

\section{Proof of Theorem 1.3}

Both amalgamated free products and HNN extensions admit a natural action on their Bass-Serre tree, yielding 1-cocycles into orthogonal representations. Very concretely, if $\Gamma=\Gamma_{1} *_{\Sigma} \Gamma_{2}$, define the orthogonal representation $\pi: \Gamma \rightarrow \mathcal{O}\left(\ell_{\mathbb{R}}^{2}(\Gamma / \Sigma)\right)$ given by left translation. Clearly $\pi$ is mixing relative to the subgroup $\Sigma$. One checks that there is a unique 1 -cocycle $b: \Gamma \rightarrow H_{\mathbb{R}}$ satisfying $b(g)=0$ for all $g \in \Gamma_{1}$ and $b(h)=\delta_{\Sigma}-\delta_{h \Sigma}$ for all $h \in \Gamma_{2}$. This 1-cocycle is unbounded and vanishes on $\Sigma$.

When $\Gamma=\operatorname{HNN}(H, \Sigma, \theta)$ is the HNN extension generated by $H$ and $t$ subject to the relations $t \sigma t^{-1}=\theta(\sigma)$ for all $\sigma \in \Sigma$, define the orthogonal representation $\pi: \Gamma \rightarrow \mathcal{O}\left(\ell_{\mathbb{R}}^{2}(\Gamma / \Sigma)\right)$ given by left translation. Again $\pi$ is mixing relative to $\Sigma$ and there is a unique 1-cocycle $b: \Gamma \rightarrow H_{\mathbb{R}}$ satisfying $b(h)=0$ for all $h \in H$ and $b(t)=\delta_{t \Sigma}$. Also this 1-cocycle is unbounded and vanishes on $\Sigma$.

So all groups $\Gamma$ appearing in Theorem 1.3 belong to $\mathcal{C} \cup \mathcal{D} \cup \mathcal{E} \cup \mathcal{C}_{2} \cup \mathcal{D}_{2}$.

Use the notations as in the formulation of Theorem 1.3. Applying Theorem 1.2 to $A:=\mathrm{M}_{n}(\mathbb{C}) \otimes$ $\mathrm{L}^{\infty}(X)$, we conclude that there exists a $\Sigma \in \mathcal{S}$ such that $\mathrm{L}^{\infty}(Y) \prec A \rtimes \Sigma$ and hence $\mathrm{L}^{\infty}(Y) \prec$ $\mathrm{L}^{\infty}(X) \rtimes \Sigma$.

Take any projection $q \in \mathrm{D}_{n}(\mathbb{C}) \otimes \mathrm{L}^{\infty}(X)$ having the same trace as $p$.

- If $\Sigma=\{e\}$, the unitary conjugacy of $\mathrm{L}^{\infty}(Y)$ and $\left(\mathrm{D}_{n}(\mathbb{C}) \otimes \mathrm{L}^{\infty}(X)\right) q$ follows from [Po01, Theorem A.1]. This settles item 2 of the theorem.

- When $\Gamma$ is a nontrivial amalgamated free product or an HNN extension and if $\Sigma$ is weakly malnormal, [HPV10, Proposition 8] provides a finite group $\Sigma_{0}$ such that $\mathrm{L}^{\infty}(Y) \prec \mathrm{L}^{\infty}(X) \rtimes \Sigma_{0}$. Then also $\mathrm{L}^{\infty}(Y) \prec \mathrm{L}^{\infty}(X)$ and the conclusion follows again from [Po01, Theorem A.1]. This settles items 3,4 and 5 of the theorem.

- If $\Sigma$ is relatively malnormal, take a subgroup $\Sigma<\Lambda<\Gamma$ such that $\Lambda<\Gamma$ has infinite index and $g \Sigma g^{-1} \cap \Sigma$ is finite for all $g \in \Gamma-\Lambda$. We apply Lemma 6.3 below. Since the normalizer of $\mathrm{L}^{\infty}(Y)$ is the whole of $p(A \rtimes \Gamma) p$ and since $\Lambda<\Gamma$ has infinite index, we conclude that $\mathrm{L}^{\infty}(Y) \prec A$ and hence $\mathrm{L}^{\infty}(Y) \prec \mathrm{L}^{\infty}(X)$. We again find the unitary conjugacy of $\mathrm{L}^{\infty}(Y)$ and $\left(\mathrm{D}_{n}(\mathbb{C}) \otimes \mathrm{L}^{\infty}(X)\right) q$ from [P001, Theorem A.1]. This settles the remaining item 1 of the theorem. 
So the proof of Theorem 1.3 is complete.

Our last lemma is implicitly contained in [Va07, Lemma 4.2], but we provide an explicit proof for the convenience of the reader.

Lemma 6.3. Let $\Gamma \curvearrowright(A, \tau)$ be any trace preserving action of a countable group. Assume that $\Sigma<\Lambda<\Gamma$ are subgroups such that $g \Sigma g^{-1} \cap \Sigma$ is finite for all $g \in \Gamma-\Lambda$. Put $M=A \rtimes \Gamma$. Let $p \in M$ be a projection and $B \subset p M p$ a von Neumann subalgebra. Denote by $Q$ the normalizer of $B$ inside $p M p$.

If $B \prec A \rtimes \Sigma$ and $B \nprec A$, then $Q \prec A \rtimes \Lambda$.

Proof. Take a nonzero partial isometry $v \in \mathrm{M}_{1, n}(\mathbb{C}) \otimes p M$ and a, possibly non-unital, normal *homomorphism $\theta: B \rightarrow \mathrm{M}_{n}(\mathbb{C}) \otimes(A \rtimes \Sigma)$ satisfying $b v=v \theta(b)$ for all $b \in B$. Put $q=\theta(p)$. Write $N=\mathrm{M}_{n}(\mathbb{C}) \otimes A$. By [Va07, Remark 3.8] we may assume that

$$
\theta(B) \nprec_{N \rtimes \Sigma} N \text {. }
$$

Whenever $\mathcal{F} \subset \Gamma$, denote by $P_{\mathcal{F}}$ the orthogonal projection onto the closed linear span of $\left\{a u_{g} \mid\right.$ $a \in N, g \in \mathcal{F}\}$. Because of 6.4 we can take a sequence of unitaries $b_{n} \in \mathcal{U}(B)$ such that $\left\|P_{\mathcal{F}}\left(\theta\left(b_{n}\right)\right)\right\|_{2} \rightarrow 0$ for every finite subset $\mathcal{F} \subset \Sigma$.

We claim that $\left\|E_{N \rtimes \Sigma}\left(x \theta\left(b_{n}\right) y\right)\right\|_{2} \rightarrow 0$ whenever $x, y \in(N \rtimes \Gamma) \ominus(N \rtimes \Lambda)$. Since we can approximate $x$ and $y$ by linear combinations of $a u_{g}, a \in N$ and $g \in \Gamma-\Lambda$, it suffices to prove the claim when $x=u_{g}, y=u_{h}$ for some $g, h \in \Gamma-\Lambda$. But then

$$
E_{N \rtimes \Sigma}\left(u_{g} \theta\left(b_{n}\right) u_{h}\right)=u_{g} P_{\Sigma \cap g^{-1} \Sigma h^{-1}}\left(\theta\left(b_{n}\right)\right) u_{h} .
$$

The claim follows from the fact that $\Sigma \cap g^{-1} \Sigma h^{-1}$ is finite.

We prove that $v^{*} Q v \subset N \rtimes \Lambda$, so that in particular, $Q \prec A \rtimes \Lambda$. Take $d \in \mathcal{N}_{p M p}(B)$. We have to prove that $v^{*} d v \in N \rtimes \Lambda$. Write $x=v^{*} d v-E_{N \rtimes \Lambda}\left(v^{*} d v\right)$. We have to prove that $x=0$. By construction, $x \theta\left(b_{n}\right) x^{*}=\theta\left(d b_{n} d^{*}\right) x x^{*}$. Hence,

$$
\left\|E_{N \rtimes \Sigma}\left(x x^{*}\right)\right\|_{2}=\left\|\theta\left(d b_{n} d^{*}\right) E_{N \rtimes \Sigma}\left(x x^{*}\right)\right\|_{2}=\left\|E_{N \rtimes \Sigma}\left(x \theta\left(b_{n}\right) x^{*}\right)\right\|_{2} \rightarrow 0
$$

by the claim in the previous paragraph. So, $x=0$ and the lemma is proven.

\section{Proof of Theorem 1.4}

Assume that $B \subset\left(\mathrm{L}^{\infty}(X) \rtimes \Gamma\right)^{t}$ is a group measure space Cartan subalgebra. Literally repeating the proof of Theorem 1.3 it follows that $B \prec \mathrm{L}^{\infty}(X)$. By [OP07, Lemma 4.11] the action $\Gamma \curvearrowright(X, \mu)$ is essentially free, contradicting the assumptions of Theorem 1.4 .

\section{References}

[BO08] N. P. Brown and N. Ozawa, C $\mathrm{C}^{*}$-algebras and finite-dimensional approximations. Graduate Studies in Mathematics 88. American Mathematical Society, Providence, 2008.

[CP10] I. Chifan and J. Peterson, Some unique group-measure space decomposition results. Preprint. arXiv: 1010.5194

[CFW81] A. Connes, J. Feldman and B. Weiss, An amenable equivalence relation is generated by a single transformation. Ergodic Theory Dynam. Systems 1 (1981), 431-450. 
[CTV06] Y. de Cornulier, R. Tessera and A. Valette, Isometric group actions on Banach spaces and representations vanishing at infinity. Transform. Groups 13 (2008), 125-147.

[CSV07] Y. de Cornulier, Y. Stalder and A. Valette, Proper actions of lamplighter groups associated with free groups. C.R. Acad. Sci. Paris, Ser. I 346 (2008), 173-176.

[FV10] P. Fima and S. Vaes, HNN extensions and unique group measure space decomposition of $\mathrm{II}_{1}$ factors. Trans. Amer. Math. Soc., to appear. arXiv:1005.5002

[HPV10] C. Houdayer, S. Popa and S. Vaes, A class of groups for which every action is $\mathrm{W}^{*}$-superrigid. Groups Geom. Dyn., to appear. arXiv:1010.5077

[IPV10] A. Ioana, S. Popa and S. Vaes, A class of superrigid group von Neumann algebras. Preprint. arXiv: 1007.1412

[Ki09] Y. Kida, Rigidity of amalgamated free products in measure equivalence theory. To appear in $J$. Topol. arXiv:0902.2888

[Ki10] Y. Kida, Examples of amalgamated free products and coupling rigidity. Ergodic Theory Dynam. Systems, to appear. arXiv:1007.1529

[MS02] N. Monod and Y. Shalom, Orbit equivalence rigidity and bounded cohomology. Ann. Math. 164 (2006), 825-878.

[Oz03] N. Ozawa, Solid von Neumann algebras. Acta Math. 192 (2004), 111-117.

[Oz04] N. Ozawa, A Kurosh-type theorem for type $\mathrm{II}_{1}$ factors. Int. Math. Res. Not. 2006, Art. ID 97560.

[OP07] N. Ozawa and S. Popa, On a class of $\mathrm{II}_{1}$ factors with at most one Cartan subalgebra. Ann. Math. 172 (2010), 713-749.

[OP08] N. Ozawa and S. Popa, On a class of $\mathrm{II}_{1}$ factors with at most one Cartan subalgebra, II. Amer. J. Math. 132 (2010), 841-866.

[Pe06] J. Peterson, $L^{2}$-rigidity in von Neumann algebras. Invent. Math. 175 (2009), 417-433.

[Pe09] J. Peterson, Examples of group actions which are virtually $\mathrm{W}^{*}$-superrigid. Preprint. arXiv: 1002.1745

[Po01] S. Popa, On a class of type $\mathrm{II}_{1}$ factors with Betti numbers invariants. Ann. of Math. 163 (2006), 809-899.

[Po03] S. Popa, Strong rigidity of $\mathrm{II}_{1}$ factors arising from malleable actions of $w$-rigid groups, I. Invent. Math. 165 (2006), 369-408.

[Po05] S. Popa, Cocycle and orbit equivalence superrigidity for malleable actions of $w$-rigid groups. Invent. Math. 170 (2007), 243-295.

[Po06a] S. Popa, Deformation and rigidity for group actions and von Neumann algebras. In Proceedings of the International Congress of Mathematicians (Madrid, 2006), Vol. I, European Mathematical Society Publishing House, 2007, p. 445-477.

[Po06b] S. Popa, On Ozawa's Property for Free Group Factors. Int. Math. Res. Not. 2007, Article ID rnm036.

[Po06c] S. Popa, On the superrigidity of malleable actions with spectral gap. J. Amer. Math. Soc. 21 (2008), 981-1000.

[PV09] S. Popa and S. Vaes, Group measure space decomposition of $\mathrm{II}_{1}$ factors and $\mathrm{W}^{*}$-superrigidity. Invent. Math. 182 (2010), 371-417.

[Si10] T. Sinclair, Strong solidity of group factors from lattices in $\mathrm{SO}(n, 1)$ and $\mathrm{SU}(n, 1)$. J. Funct. Anal. 260 (2011), 3209-3221.

[Va07] S. Vaes, Explicit computations of all finite index bimodules for a family of $\mathrm{II}_{1}$ factors. Ann. Sci. École Norm. Sup. 41 (2008), 743-788.

[Va10] S. Vaes, Rigidity for von Neumann algebras and their invariants. In Proceedings of the International Congress of Mathematicians (Hyderabad, 2010), Vol. III, Hindustan Book Agency, 2010, p. 16241650. 